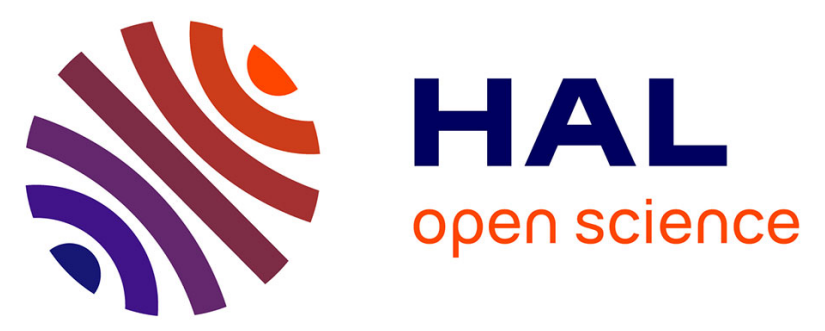

\title{
Fingerprinting and relocating tectonic slices along the plate interface: Evidence from the Lago Superiore unit at Monviso (Western Alps)
}

Mattia Gilio, Marco Scambelluri, Samuele Agostini, Marguerite Godard, Thomas Pettke, Philippe Agard, Michele Locatelli, Samuel Angiboust

\section{To cite this version:}

Mattia Gilio, Marco Scambelluri, Samuele Agostini, Marguerite Godard, Thomas Pettke, et al.. Fingerprinting and relocating tectonic slices along the plate interface: Evidence from the Lago Superiore unit at Monviso (Western Alps). Lithos, 2020, 352-353, pp.105308. 10.1016/j.lithos.2019.105308 . hal-02510337

\section{HAL Id: hal-02510337 \\ https://hal.umontpellier.fr/hal-02510337}

Submitted on 9 Nov 2020

HAL is a multi-disciplinary open access archive for the deposit and dissemination of scientific research documents, whether they are published or not. The documents may come from teaching and research institutions in France or abroad, or from public or private research centers.
L'archive ouverte pluridisciplinaire $\mathbf{H A L}$, est destinée au dépôt et à la diffusion de documents scientifiques de niveau recherche, publiés ou non, émanant des établissements d'enseignement et de recherche français ou étrangers, des laboratoires publics ou privés. 


\title{
Fingerprinting and relocating tectonic slices along the plate interface: Evidence from the Lago Superiore unit at Monviso (Western Alps)
}

\author{
Mattia Gilio a,b,*, Marco Scambelluri a , Samuele Agostini ${ }^{c}$, Marguerite Godard ${ }^{\mathrm{d}}$, Thomas Pettke ${ }^{\mathrm{e}}$, \\ Philippe Agard ${ }^{\mathrm{f}}$, Michele Locatelli ${ }^{\mathrm{f}}$, Samuel Angiboust ${ }^{\mathrm{g}}$ \\ a Dipartimento di Scienze della Terra Ambiente e Vita, Università di Genova, Italy \\ b Dipartimento di Scienze della Terra e dell'Ambiente, Università di Pavia, Italy \\ c CNR - Istituto di Geoscienze e Georisorse, Pisa, Italy \\ d Géosciences Montpellier, CNRS, Université de Montpellier, Montpellier, France \\ e Institute of Geological Sciences, University of Bern, Switzerland \\ ${ }^{\mathrm{f}}$ Institut des Sciences de la Terre de Paris (ISTeP), Sorbonne Université, France \\ ${ }^{g}$ Institut de Physique du Globe de Paris, France
}

\begin{abstract}
A B S T R A C T
The Lago Superiore Unit (LSU, Monviso Massif, Italian Western Alps) is a section of fossil oceanic lithosphere equilibrated to eclogite facies conditions $\left(550{ }^{\circ} \mathrm{C}-2.8 \mathrm{GPa}\right)$ during Alpine subduction $(45-40 \mathrm{Ma})$. It is cut by two major shear zones, namely the Intermediate (ISZ) and Lower Shear Zone (LSZ), mostly consisting of serpentinite. The lowermost, serpentine-rich, section of the Lago Superiore Unit, the Basal Serpentinite, separates the HP ophiolite domain from the underlying continental Dora-Maira Unit.

Here we show that the LSZ and the Basal Serpentinite were active at different stages of the subduction and exhumation history of the complex. Most of retrograde deformation and mineral re-equilibration were localized in the LSZ. Channelized fluids percolating during this phase chemically homogenized the LSZ serpentinites, that preserved their HP mineralogy only locally; the best-preserved relicts of the eclogite-facies high pressure stage within the LSZ serpentinite are nodules of magnesite (representing former veins) and eclogite blocks. Differently, the underlying Basal Serpentinite largely escaped the exhumation-related processes and still records the prograde chemical and petrological history of the LSU serpentinite, from ocean-floor hydration to HP metamorphic conditions.

The Lago Superiore Unit thus represents a snapshot of major Alpine metamorphic and shearing events, from prograde subduction to exhumation. Its km-scale thickness, and the oriented antigorite fabric in the Lower Shear Zone and Basal Serpentinite makes it a good seismic reflector. This HP ophiolite complex can thus be used as proxy of a deep (70-80 km) Alpine-type subduction zone, and to better constrain and interpret seismic images of present-day convergent margins.
\end{abstract}

\section{Introduction}

Recent work shows that oceanic (abyssal) and subduction-zone serpentinites carry volatile and fluid-mobile elements (FME; B, Cs, W, $\mathrm{As}, \mathrm{Sb}, \mathrm{Be}, \mathrm{U}$ ) acquired during chemical interaction with circulating fluids (Deschamps et al., 2013; Kodolányi et al., 2012; Peters et al., 2017; Scambelluri et al., 2019; Scambelluri and Tonarini, 2012). The subduction-zone chemical processing of serpentinite mostly affects the slices of slab and of supra-subduction mantle rocks that are tectonically accreted along the interface between converging plates and are

* Corresponding author at: Dipartimento di Scienze della Terra e dell'Ambiente, Università di Pavia, Italy.

E-mail address: mattia.gilio@unipv.it (M. Gilio). flushed by fluids released by the slab crustal reservoirs (Cannaò et al., 2016; Hattori and Guillot, 2007; Lafay et al., 2013; Scambelluri et al., 2014; Scambelluri and Tonarini, 2012). The volatile and FME budgets of oceanic and of subduction-zone serpentinite can be released to metamorphic fluids produced by a series of serpentinite dehydration reactions during burial to forearc and sub-arc depths (Deschamps et al., 2013; Scambelluri et al., 2004, 2015). Tectonic slicing of oceanic slabs along the subduction interface (Agard et al., 2009; Agard et al., 2018; Ruh et al., 2015) and their deformation-enhanced chemical interaction with slab fluids thus affects the geochemical cycling of volatile and FME in subduction zones and, consequently, mantle metasomatism and arc magmatism (Angiboust et al., 2012c; Bebout, 2007; Ribeiro and Lee, 2017; Scambelluri and Tonarini, 2012). Understanding the formation mechanisms and the architecture of plate interface domains, 
together with their tectonic and chemical processing is thus of primary interest to interpret fossil and present-day evolutionary aspects of subduction zones (Agard et al., 2018; Bebout, 2007; Breeding et al., 2004).

Much knowledge of the interface domains between convergent plates arises from seismic tomography showing that they correspond to 2-8 km-thick layers atop the subducting slab characterized by low velocity of P and S seismic waves (Bostock et al., 2002; Van Keken et al., 2002; Wada and Wang, 2009). Recent numerical modelling and field-based studies of exhumed high-pressure (HP) ophiolite complexes assessed that serpentine-bearing slices can be tectonically accreted atop the crustal and sedimentary sequences of the downgoing oceanic plate (Angiboust et al., 2012c; Cannaò et al., 2016; Gerya et al., 2002; Schwartz et al., 2001). This tectonic movement is not only accompanied by slab fluid influx (Cannaò et al., 2016) but also by seismic activity (as proposed by Angiboust et al., 2012a). However, the resolution of geophysical data does not enable to image the main structures accomplishing tectonic accretion of slab and mantle wedge slices at the plate interface, so to define the architecture and rock composition of such settings. Structural, petrological and geochemical studies of fossil subduction environments are crucial to define the above features and to assessing the mechanisms of coupling/decoupling of oceanic (ophiolitic) slices in the subduction interface (Agard et al., 2009, 2018).

Here we present the petrological and geochemical study of two adjacent serpentinite shear zones, the Lower Shear Zone (LZS) and the Basal Serpentinite from the Lago Superiore Unit (LSU; Fig. 1a) of the Monviso Massif (Western Italian Alps). This is a well preserved section of fossil oceanic lithosphere involved in Alpine subduction and key example of high-pressure ophiolite detached from the down-going oceanic slab at $80 \mathrm{~km}$ depths and tectonically accreted to plate interface settings (Angiboust et al., 2011; Angiboust et al., 2012b; Locatelli et al., 2018, 2019; Lombardo et al., 1978). Our aim is to characterize the pressure-temperature conditions at the time of shear zone activity, the extent of fluid-rock interaction in serpentinites, and the timing of serpentinite coupling/accretion along the subduction interface.

\section{Geologic background}

The Monviso metaophiolite Massif is located in the Western European Alps (Fig. 1) and crops out along the French-Italian border for $\sim 30 \mathrm{~km}$ (Lombardo et al., 1978). It belongs to the Jurassic Ligurian Piemontese oceanic domain and was involved in Alpine subduction about 46 million years ago (Agard et al., 2009; Lombardo et al., 2002; Rubatto and Hermann, 2003). It consists of ophiolitic units made of calcschist, pillow lava, metagabbros, and serpentinite (Fig. 1a-b) formed in the Jurassic Ligurian Tethys and later involved in the Alpine subduction and exhumation orogenic cycle (Lombardo et al., 1978). Successive field and petrologic studies performed on the Monviso Massif have refined interpretations of its tectonic structure, from a set of tectonic units showing different peak metamorphic conditions and assembled in the so called 'subduction channel' (Guillot et al., 2004) to the existence of two coherent oceanic units separated by west-dipping shear zones (Angiboust et al., 2012b; Angiboust et al., 2014; Lombardo et al.,
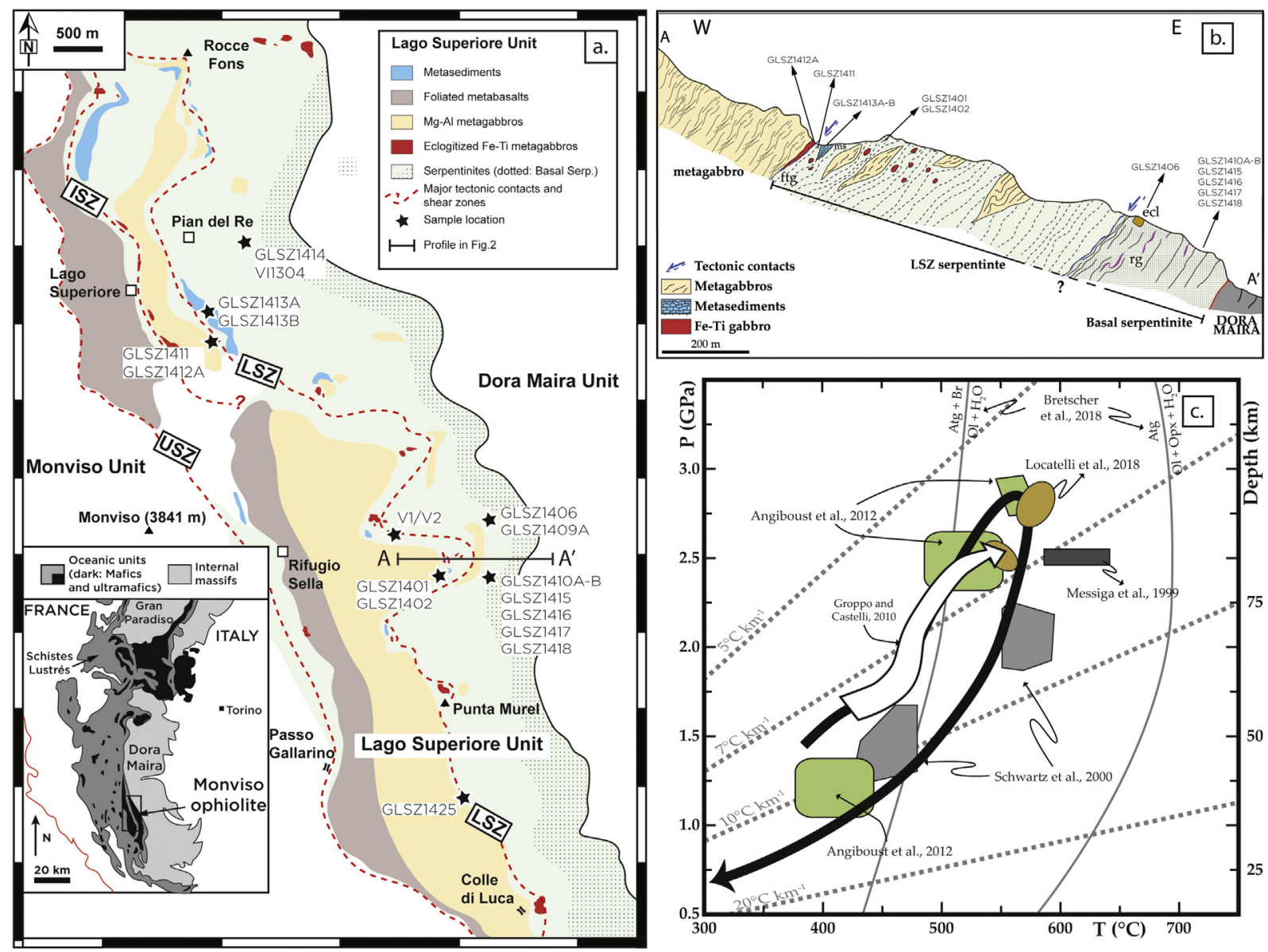

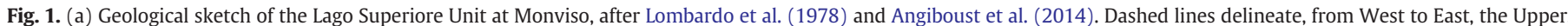

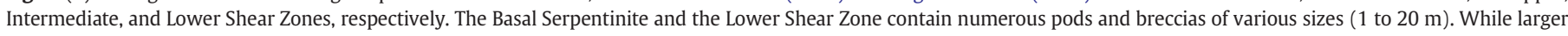

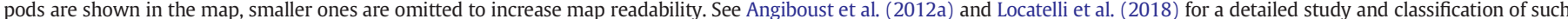

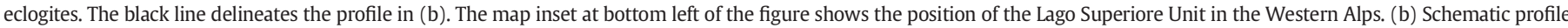

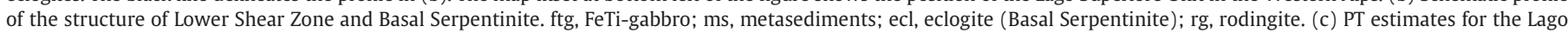
Superiore Unit at Monviso. The black arrow summarizes the PT path of the Lago Superiore Unit (Angiboust et al., 2014). Antigorite dehydration reactions after Bretscher et al., 2018. 
1978). Balestro et al. (2015) and Festa et al. (2015) also proposed that the Monviso metaophiolite partly derives from an oceanic core complex and preserves some original geometries and lithological relationships despite the subduction and exhumation cycle, similar to what has been observed in the Alpine Corsica (Brovarone et al., 2011).

Following the early the work by Lombardo et al. (1978), thermobarometric estimates on mafic eclogites resulted in contrasting peak P-T conditions from 450 to $650{ }^{\circ} \mathrm{C}$ and from 1.2 to $2.4 \mathrm{GPa}$ (Fig. 1c; Blake Jr et al., 1995; Messiga et al., 1999; Schwartz et al., 2000; Castelli et al., 2002; Groppo and Castelli, 2010). Angiboust et al. (2012b) suggested that this difference stems (i) from comparing different methods with large uncertainties, (ii) from poorly constrained mineral and bulk rock $\mathrm{Fe}^{3+}$ and (iii) from prominent variations in bulk-rock composition. They performed new geothermobarometric estimates for the Monviso area and identified two main tectonic units based on structural and petrologic criteria. These are the Monviso Unit s.s., equilibrated at $480-500{ }^{\circ} \mathrm{C}$ and $2.1-2.2 \mathrm{GPa}$, and the Lago Superiore Unit, that experienced homogeneous eclogite facies conditions at higher P-T conditions of $530-550{ }^{\circ} \mathrm{C}$ and $2.6-2.8 \mathrm{GPa}$ (see also Locatelli et al., 2018). Balestro et al. (2014) performed a similar subdivision of the Monviso metaophiolite into Upper and Lower Tectonic Unit (i.e. Monviso Unit s.s. and Lago Superiore Unit respectively). In this work, we employ subdivisions and nomenclature from Angiboust et al. (2012b) and Locatelli et al., 2018.

According to the recent work by Angiboust et al. (2012b), Angiboust et al. (2014), Locatelli et al. (2018) and Locatelli et al. (2019), the two main tectonic units composing the Monviso Massif record distinct structural and petrological histories and are separated by a series of 1-50 m thick mylonitic serpentinite slivers (Fig. 1a-b). The Monviso Unit includes the classical Vallanta and Costa Ticino units defined by Lombardo et al. (1978) and represents an overturned sequence of $\mathrm{Mg}$ - Al gabbros overlying pillow basalts and metasediments (Angiboust et al., 2012b). The Lago Superiore Unit (Fig. 1a) consists, from top to bottom, of (1) calcschists and metabasalts, (2) a variably thick sequence of metagabbros cross-cut by $\mathrm{Fe}-\mathrm{Ti}$ gabbros, and (3) serpentinite hosting $\mathrm{Mg}-\mathrm{Al}$ and $\mathrm{Fe}-\mathrm{Ti}$ eclogitic metagabbros, plagiogranite (Castelli et al., 2002; Castelli and Lombardo, 2007) and jadeitite (Compagnoni et al., 2007). It is cut by a shear zone (the Lower Shear Zone, LSZ) that separates the above sequence from a 300 m-thick Basal Serpentinite layer (Fig. 1a-b; Guillot et al., 2004; Auzende et al., 2006). The Lago Superiore Unit underwent eclogitization and intense deformation during the Alpine subduction event (Angiboust et al., 2012b, 2014; Groppo and Castelli, 2010; Locatelli et al., 2018; Locatelli et al., 2019; Lombardo et al., 1978).

A serpentine-rich shear zone, referred to as the Upper Shear Zone (USZ; Fig. 1a), divides the Lago Superiore Unit from the Monviso Unit (Angiboust et al., 2014). Moreover, two eclogite-facies shear zones, the Intermediate Shear Zone (ISZ) and the Lower Shear Zone (LSZ; Fig. 1a), cross-cut the Lago Superiore Unit (Angiboust et al., 2014). Within the ISZ, Fe-Ti metagabbros underwent eclogite facies deformation forming planar omphacite + garnet fabrics (Philippot and van Roermund, 1992) and HP omphacite crack-seal veins (Philippot and Kienast, 1989; Philippot and Selverstone, 1991; Spandler et al., 2011). The LSZ (which also includes the Baracun Shear Zone described in Balestro et al., 2015, 2018) defines the contact between the Lago Superiore Unit and the Basal Serpentinite, which in turn lies on top of the Dora-Maira massif. It consists of deformed eclogite, eclogite breccias (Angiboust et al., 2012a), metagabbros and metasediments enclosed in a strongly foliated serpentinite matrix and was affected by mass exchange, metasomatism and fluid flux during eclogite facies conditions and exhumation (Angiboust et al., 2014).

Uranium-Pb zircon dating of the Monviso meta-igneous rocks yields late-Jurassic oceanic crystallization ages throughout the entire unit (152 \pm 2 Ma, Lombardo et al., 2002; $163 \pm 2$ Ma, Rubatto and Hermann, 2003). This suggests that the entire intrusive sequence at Monviso formed within 10 Myrs, during Callovian to Kimmeridgian times (Lombardo et al., 1978). Dating the Alpine eclogitic event yielded contrasting results. Cliff et al. (1998) reported Sm-Nd ages of $60 \pm 12$ and $62 \pm 9 \mathrm{Ma}$, whereas Duchêne et al. (1997) reported Lu—Hf ages of $49.2 \pm 1.2 \mathrm{Ma}$. Monié (1989) achieved Ar/Ar ages of 48-50 Ma, which are potentially affected by excess argon as suggested by Rubatto and Hermann (2003). More recent dating of metamorphic zircon inclusions inside an omphacite vein yielded an age of $45.0 \pm 1.0 \mathrm{Ma}$ (Rubatto and Hermann, 2003), consistent with the $\mathrm{Rb}-\mathrm{Sr}$ age on phengite of $40 \pm$ 1.0 Ma (cooling age to below $500{ }^{\circ} \mathrm{C}$ ) reported by (Cliff et al., 1998).

\section{Analytical methods}

The main analytical methods are only briefly described here and further detailed in the Repository Database.

Bulk rock major element concentrations were measured either by $\mathrm{XRF}$ at the Activation Laboratories in Toronto, Canada, or by the laserablation ICP-MS pressed powder pellet (LA-ICP-MS PPP) technique at the University of Bern, Switzerland (Peters and Pettke, 2017). Trace element measurements were done either by liquid-mode ICP-MS at the University of Montpellier (F) following the procedures described in Ionov et al. (1992) and in Godard et al. (2000) or by LA-ICP-MS PPP at the University of Bern, Switzerland. Strontium and $\mathrm{Pb}$ isotope ratio measurements were performed using a Finnigan MAT 262 multiple collector thermal ionization mass spectrometer (TIMS) at IGG-CNR of Pisa (Italy). Strontium ratios are reported with age correction (45 Ma; Rubatto and Hermann, 2003) using $\mathrm{Rb}$ and $\mathrm{Sr}$ concentrations obtained from liquid mode ICP-MS measurements, respectively. Lead isotopic ratios were not corrected for their age due to very low $\mathrm{U} / \mathrm{Pb}$ ratios measured in serpentinite.

In-situ major element $\left(\mathrm{SiO}_{2}, \mathrm{TiO}_{2}, \mathrm{Al}_{2} \mathrm{O}_{3}, \mathrm{Cr}_{2} \mathrm{O}_{3}, \mathrm{FeO}, \mathrm{Fe}_{2} \mathrm{O}_{3}\right.$ estimated from stoichiometries, $\mathrm{MgO}, \mathrm{MnO}, \mathrm{CaO}, \mathrm{NiO}, \mathrm{Na}_{2} \mathrm{O}$, and $\mathrm{K}_{2} \mathrm{O}$ ) compositions of minerals were measured using a JEOL JXA 8200 Superprobe equipped with five wavelengths-dispersive (WDS) spectrometers, an energy dispersive (EDS) spectrometer, and a cathodoluminescence detector (accelerating potential $15 \mathrm{kV}$, beam current $15 \mathrm{nA}$ ), operating at the Dipartimento di Scienze della Terra, Università di Milano.

\section{Field Occurrence of the investigated samples}

The LSZ is a variably thick (40-150 m) and $5 \mathrm{~km}$ long highly deformed west-dipping serpentinite zone which separates the main section of the Lago Superiore Unit (metagabbros and metabasalts) from the Basal Serpentinite (Fig. 1a-b). It consists of strongly foliated antigorite serpentinite (Fig. 2a) and local chlorite-schists embedding magnesite nodules (Fig. 2b) and metagabbro blocks of various typology and dimension. Such metagabbro blocks range from mylonite to breccias and belong to eclogitic $\mathrm{Mg}-\mathrm{Al}$ and $\mathrm{Fe}-\mathrm{Ti}$ metagabbro bodies of the LSU, cropping out at the top of the LSZ (Fig. 1a-b). Locatelli et al. (2018) performed a detailed statistical study of the occurrence and fabric of such blocks, distinguishing three main types: (1) brecciated $\mathrm{Fe}-\mathrm{Ti}$ gabbros near the upper section of the LSZ; (2) unbrecciated decametric slivers of $\mathrm{Mg}$ - Al gabbros; and (3) unbrecciated $\mathrm{Fe}-\mathrm{Ti}$ gabbros, located towards the bottom of the LSZ.

The Basal Serpentinite consists of strongly foliated olivine-antigorite serpentinite with decimeter-thick, boudinaged rodingite dykes and subordinate eclogite, generally found along the top boundary Fig. 2c. The foliated Basal Serpentinite embodies some little deformed domains (e.g. Pian del Re area; Fig. 1) showing a well-preserved mantle texture and clinopyroxene relicts. In the undeformed serpentinite, the mantle olivine and pyroxene sites are still recognizable due to their characteristic replacement texture consisting of mesh antigorite + magnetite after primary mantle olivine and of pseudomorphic antigorite after primary mantle clinopyroxene. Most eclogite blocks in the Basal Serpentinite preserve the original magmatic fabric, however some eclogite $\mathrm{Fe}-\mathrm{Ti}$ gabbroic pods, more numerous in the upper portion of the Basal Serpentinite, show no difference to the Fe-Ti breccias (except that 


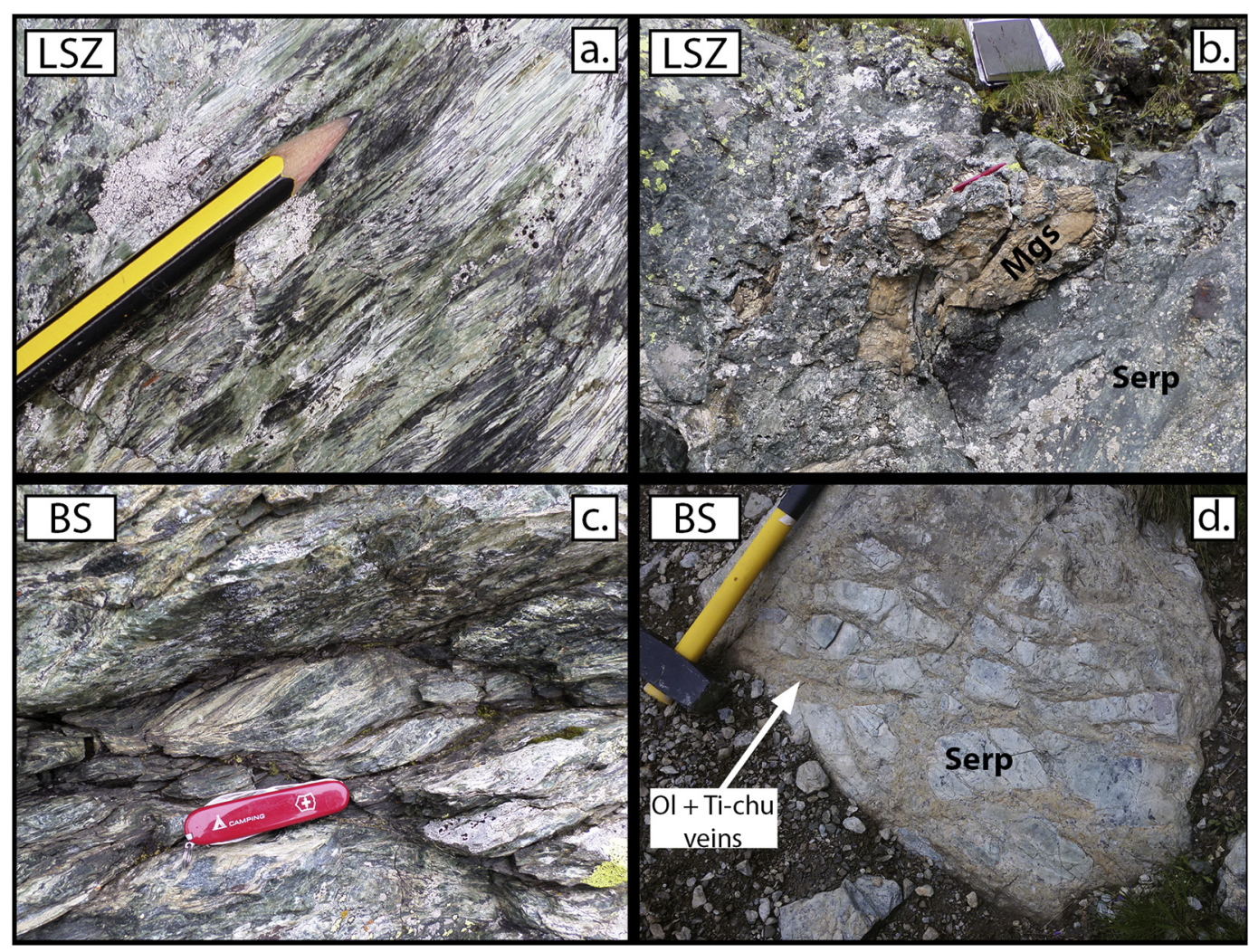

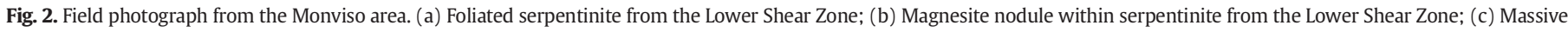
serpentinite from the Basal Serpentinite; (d) Olivine + Ti-clinohumite + magnetite veins cutting through a massive serpentinite from the Basal Serpentinite.

they are not brecciated) nor to the unbrecciated blocks (type 3; Locatelli et al., 2018) found at the base of the LSZ. A peculiar feature of the Basal Serpentinite is the widespread occurrence of $1-2 \mathrm{~cm}$ thick olivine + Ticlinohumite + magnetite veins (Fig. 2d), generally cutting the massive serpentinite domains or aligned parallel or sub-parallel to the highpressure (HP) olivine + antigorite foliation.

The boundary between the LSZ into the Basal Serpentinite is gradual and generally ascribed to an arbitrary divide between serpentine schists (LSZ) and a more competent serpentinite associated with olivine veins and metarodingite (Angiboust et al., 2014; Locatelli et al., 2018). To the contrast, the lower contact of the Basal Serpentinite with the
Dora-Maira Massif consists of a sharp lithological boundary where the serpentinite shows a pervasive foliation defined by a 5-10 m thick olivine-free antigorite serpentinite.

In this work, we sampled eclogite, serpentinite and metasedimentary rocks along two cross-sections from the top of the LSZ to the bottom of the Basal Serpentinite, near the contact with the Dora-Maria Massif (Fig. 1b). The main collected samples (names GLSZ14- are abbreviated to \#), their location and mineralogy are summarized in Table 1. In the LSZ, we collected four antigorite serpentinites (\#01, \#02,\#11,\#25), one eclogite (\#12A), two metasediments (\#13A, \#13B) and two magnesite veins in serpentinite (V1, V2). In the Basal

Table 1

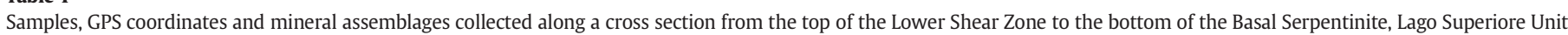
(Monviso ophiolite). GPS coordinate system: WGS84 - UMT zone $32 \mathrm{~N}$.

\begin{tabular}{|c|c|c|c|c|c|}
\hline & \multirow[t]{2}{*}{ Sample name } & \multirow[t]{2}{*}{ Rock type } & \multirow[t]{2}{*}{ Mineral assemblage } & \multicolumn{2}{|c|}{ GPS coordinates } \\
\hline & & & & North & East \\
\hline \multirow[t]{8}{*}{ LSZ } & GLSZ1401 & Foliated serp. & Atg, Mt., Chl & $4,947,261$ & 351,955 \\
\hline & GLSZ1402 & Foliated serp. & Atg, Mt., Chl & $4,947,261$ & 351,955 \\
\hline & GLSZ1411 & Foliated serp. & Atg, Mt., Chl & $4,950,153$ & 349,289 \\
\hline & GLSZ1412A & Eclogite & Grt, Omph, Rt & $4,950,153$ & 349,289 \\
\hline & GLSZ1413A & Calcschist & Cal, Qtz, Wmca & $4,947,187$ & 351,849 \\
\hline & GLSZ1413B & Quartzite & Qtz, Ep, Wmca, Gr & $4,947,187$ & 351,849 \\
\hline & GLSZ1425 & Foliated serp. & Atg, Mt., Chl & $4,944,540$ & 352,221 \\
\hline & V1-V2 & MgsVein & Mgs, Tlc, Atg & n.m. & n.m. \\
\hline \multirow[t]{11}{*}{ Basal serpentinite } & GLSZ1406 & Eclogite & Grt, Omph, Rt & $4,948,021$ & 352,466 \\
\hline & GLSZ1408 & Foliated serp. with mantle relics & Atg, Chl, Mt., Aug & $4,948,089$ & 352,470 \\
\hline & GLSZ1409A & Foliated serp. with mantle relics & Atg, Chl, Mt., Aug & $4,948,089$ & 352,470 \\
\hline & GLSZ1410A & Foliated serp. with mantle relics & Atg, Chl, Mt., Aug & $4,948,089$ & 352,470 \\
\hline & GLSZ1410B & Olivine vein & Ol, Atg, Chl, Di & $4,947,176$ & 352,565 \\
\hline & GLSZ1414 & Diopside vein & Di, Mt., Atg, Chl & $4,951,498$ & 349,838 \\
\hline & GLSZ1415 & Foliated serp. & Atg, Chl, Mt & $4,945,233$ & 351,892 \\
\hline & GLSZ1416 & Ti-cl Vein & Ti-Cho/Cl, Di, Ol, Mt., Chl, Atg & $4,945,233$ & 351,892 \\
\hline & GLSZ1417 & HP Serpentinite & Ol, Atg, Chl, Di, Cal & $4,947,020$ & 352,444 \\
\hline & GLSZ1418 & Ti-cl Vein & Ti-Cho/Cl, Di, Ol, Mt., Chl, Atg & $4,946,950$ & 352,435 \\
\hline & VI1304 & Serp. with bastite and mesh structures & Serp, Chl, Mt & $4,951,498$ & 349,838 \\
\hline
\end{tabular}




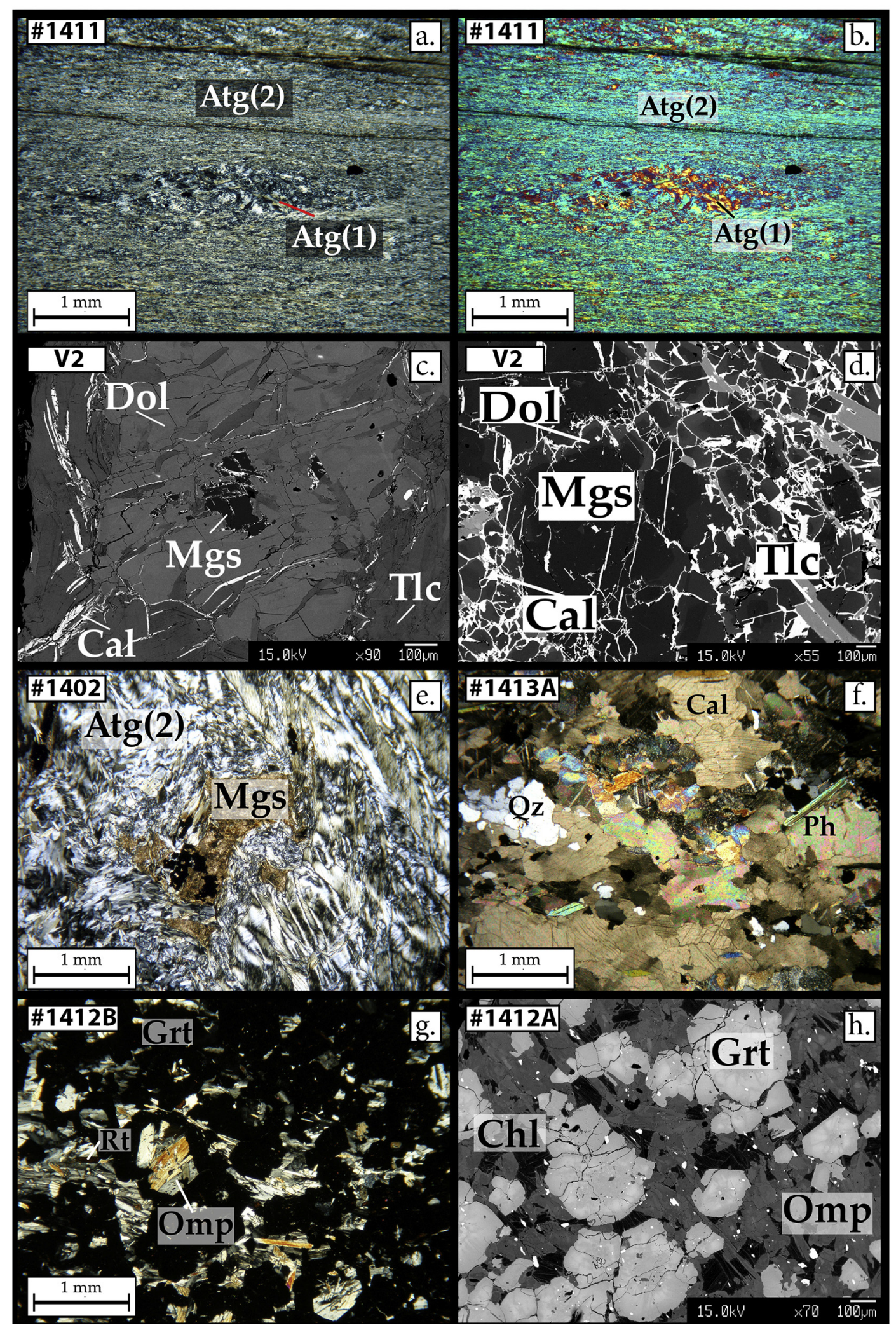

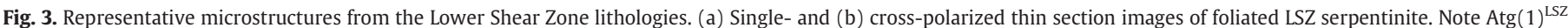

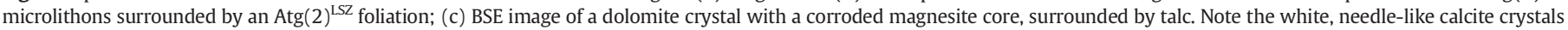

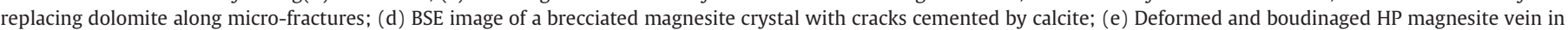

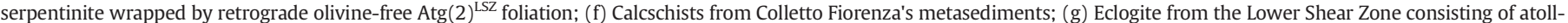

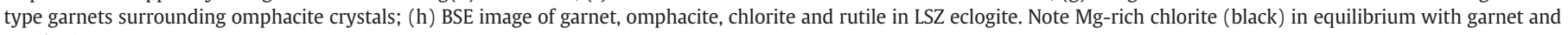
omphacite. 
Serpentinite, we collected one eclogite (\#06), six serpentinites (\#09A, $\# 10 A, \# 14, \# 15$, \#17, VI13-04), one olivine vein (\#10B) and two olivine + Ti-clinohumite veins (\#16,\#18).

\section{Petrography and microstructure}

\subsection{Lower shear zone}

\subsubsection{Serpentinite}

Serpentinites (\#01, \#11, \#25) mainly consist of antigorite (Atg), chlorite (Chl) and rare magnetite (Mag) mylonites (Fig. 3a-b). Two generations of antigorite can be recognized: $\operatorname{Atg}(1)^{\mathrm{LSZ}}$ microlithons are wrapped by tiny spaced (10-20 $\mu \mathrm{m}$; Fig. 3a-b) Atg $(2)^{\mathrm{LSZ}}$ lying along the mylonite-foliation, which is locally crenulated (\#02). Five to ten mm-sized domains with magnesite (Mgs), talc (Tal) and dolomite (Dol) are stretched along the $\operatorname{Atg}(2)^{\mathrm{LSZ}}$ foliation (Fig. 3c-d-e) and may represent former veins. Magnesite forms up-to-1-cm large porphyroclasts. Talc inclusions in magnesite display regular grain boundaries, suggesting magnesite and talc coexisted at the same P-T conditions. Magnesite locally re-crystallizes into smaller, mm-sized secondary dolomite. Dolomite also occurs in large crystals, epitaxially replacing the original magnesite, whose remnants are still preserved in the dolomite cores (Fig. 3c). In a few cases, magnesite and the secondary dolomite are brecciated and cemented by late-stage calcite (Cal; Fig. 3d). Neither metamorphic olivine (Ol), Ti-clinohumite (Ti-Chu) and diopside (Di), nor clasts of mantle minerals, have been found in LSZ serpentinites.

\subsubsection{Metasediments}

We have sampled a large metasediment sliver (20-30 m-thick) consisting of quartzite (\#13B) and calcschist (\#13A; Fig. 1a) cropping out near Colletto Fiorenza (Fig. 1a). The calcschist (Fig. 3f) consists of calcite, quartz (Qz), white mica, and epidote (Ep). The quartzite consists of coarse quartz(1), epidote(1) and white mica(1) porphyroclasts, stretched along the foliation and locally recrystallized to smaller grains, i.e. quartz(2), epidote(2), and white mica(2). Cm-sized domains of epidote and white mica including a pervasive graphitic foliation can be regarded as lawsonite pseudomorphs (Angiboust et al., 2012b; Groppo and Castelli, 2010).

\subsubsection{Eclogite}

The eclogite from Colletto Fiorenza (\#12A) consists of garnet (Grt), omphacite (Omp), rutile (Rt), and chlorite (Fig. 3g-h). It displays a layered structure with alternating omphacite- and garnet-rich domains, broadly parallel to the mylonitic foliation of serpentinites. Rutile is equally distributed in both domains. Omphacite domains consist of fine-grained omphacite(2), generally oriented along the foliation, wrapping around rare larger omphacite(1) porphyroclasts and/or coarsegrained omphacite clusters. The garnet rich domains often display atoll-like structures (Fig. 3g), surrounding large omphacite(1) crystals.

\subsection{Basal serpentinite}

Sample VI13-04 (Fig. 4a) is a relatively undeformed serpentinite preserving mesh structures and bastites attributable to static serpentinization of mantle olivine and pyroxene, respectively. In some serpentinite samples (\#09A and \#10A), primary mantle clinopyroxene is also preserved (Fig. 4b). Primary mantle olivine was not found in this study but is reported by Lombardo et al. (1978). Similarly, chrysotile and/or lizardite predating antigorite growth have not been found.

The Basal Serpentinite (\#09A, \#10A, \#15) records the transition from static, undeformed serpentinite to deformed serpentinite, represented by bastite, mesh textures, and rare chlorite + magnetite pseudomorphs after spinel, stretched along an antigorite foliation. The latter (present in samples \#09A and \#10A) wraps around original, porphyroclastic mantle clinopyroxene (Fig. 4b). These samples show no metamorphic olivine growth and may be considered as records of the prograde subduction history at Monviso.

The above antigorite foliation is cut by a mylonitic, olivine-bearing foliation (sample \#17). The olivine-bearing foliation encloses microlithons of antigorite + brucite replaced by secondary olivine (Fig. 4c-d), thus showing a transition from prograde to HP metamorphic conditions. Furthermore, brucite is also found in pressure-shadows around bastite (Fig. 4d), suggesting it was stable with the HP olivine foliation.

Three types of veins have been distinguished based on the relative abundance of the dominant vein minerals: (1) diopside (\#14), (2) Tichondrodite/clinohumite veins (\#16, \#18; Fig. 4e-f), and (3) olivine (\#10B; Fig. 4g). Diopside veins consist of coarse grained diopside (1) mantle relicts, locally recrystallized into diopside(2) and chlorite. Ti-chondrodite/clinohumite veins (Fig. 4e-f) consist of Ti-chondrodite/ clinohumite, olivine, chlorite, antigorite, and magnetite. Tichondrodite forms mm-sized crystals in equilibrium with olivine(1), magnetite, and chlorite. However, Ti-chondrodite crystals are in part statically recrystallized into olivine + Ti-clinohumite (Fig. 4f). Olivine veins consist of olivine, antigorite, and magnetite (Fig. $4 \mathrm{~g}$ ). A second generation of metamorphic olivine locally replaces antigorite (Fig. 4g).

\subsubsection{Eclogite}

Mafic eclogite (\#06) consists of garnet, omphacite, and rutile (Fig. 4h). Garnet forms large (up to $5 \mathrm{~mm}$ ), idiomorphic porphyroblasts with an inclusion-rich core (mostly omphacite) and an inclusion-free rim. Omphacite is smaller $(<1 \mathrm{~mm})$ and forms the rock's matrix. Rutile is only preserved in some titanite cores. Retrograde chlorite grows after HP garnet and omphacite. This rock is granular and lacks any recognizable structures at the meso- and micro-scale that can be associated with the hosting Basal Serpentinite.

\section{Bulk rock and mineral chemistry}

\subsection{Bulk-rock Compositions}

The bulk-rock major and trace element compositions of serpentinites, metasediments, and eclogite from the Lower Shear Zone and from the Basal Serpentinite are shown in Tables 2 and 3 and in Repository Data (Repository_EMP.xlsx). Major element compositions are illustrated in Fig. 5a-b-c, and the Rare Earth Elements (REE), normalized to CI chondrite (McDonough and Sun, 1995), and Trace Element (TE), normalized to the primitive mantle (McDonough and Sun, 1995) in Figs. 6 and 7, respectively.

\subsubsection{Major elements}

The LSZ serpentinites display a rather homogeneous composition (light green dots in Fig. 5a-b-c). Data show a depletion in $\mathrm{CaO}$ (1.2 to $0.02 \mathrm{wt} \%$ ) with respect to $\mathrm{Al}_{2} \mathrm{O}_{3}(1.2-3.0 \mathrm{wt} \%$, Fig. 5a). These low abundances of $\mathrm{CaO}$ reflect partial lost during seafloor hydration, due to LT serpentinization of mantle diopside. The $\mathrm{FeO}$ and $\mathrm{MgO}$ compositions of LSZ serpentinite (Fig. 5b) are rather homogeneous, with $\mathrm{FeO}=$ 7.4-8.3 (note that in Table $2 \mathrm{FeO}$ is expressed as $\mathrm{Fe}_{2} \mathrm{O}_{3}^{\mathrm{T}}$, representing total iron expressed as $\mathrm{Fe}_{2} \mathrm{O}_{3}$ in absence of measured $\mathrm{FeO}$ and $\mathrm{Fe}_{2} \mathrm{O}_{3}$ compositions) and $\mathrm{MgO}=36.3-36.7$. These very similar $\mathrm{Fe}-\mathrm{Mg}$ values indicate that the LSZ was a homogenous peridotite of harzburgitic composition, at least regarding the major element composition. The magnesite vein hosted in serpentinite show the composition of a ternary carbonate with loss on ignition (L.O.I.) $=46 \mathrm{wt} \%$ and $\mathrm{MgO}, \mathrm{FeO}$ and $\mathrm{CaO} 34.7,10.0$ and $5.2 \mathrm{wt} \%$, respectively (Table 2). The $\mathrm{SiO}_{2}$ content in the vein is about $1.1 \mathrm{wt} \%$, possibly reflecting talc inclusions in magnesite.

The LSZ metasediments include quartz-micaschists and calcschists. Quartz-micaschists (\#13B) have $\mathrm{SiO}_{2}=57.8 \mathrm{wt} \%$ and $\mathrm{Al}_{2} \mathrm{O}_{3}$ of $20.8 \mathrm{wt}$ $\%$. The calcschist layers (\#13A) display $42.4 \mathrm{wt} \% \mathrm{CaO}$ and a L.O.I. = $34 \mathrm{wt} \%$. The composition of the LSZ eclogite (\#12A) is close to the one 


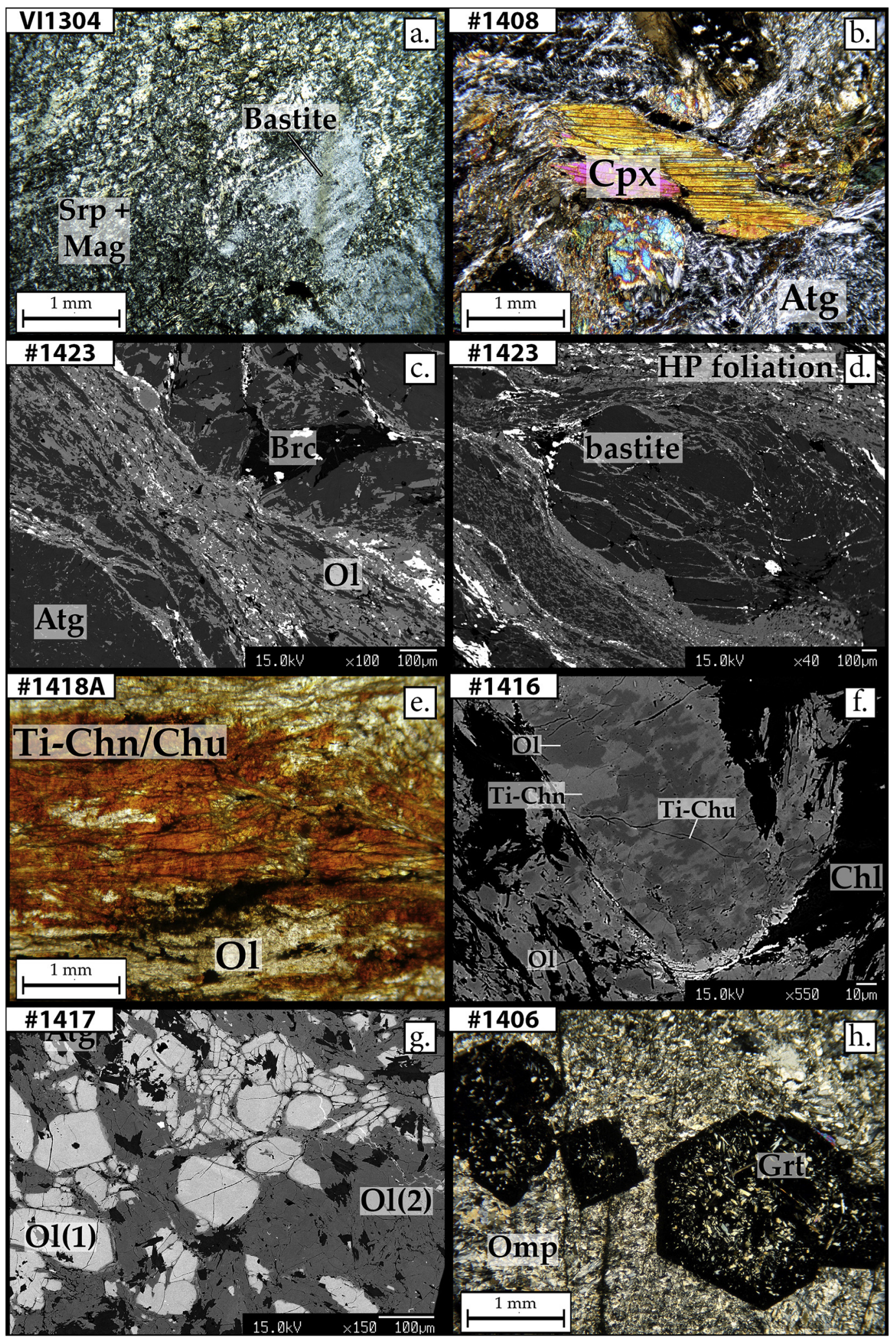

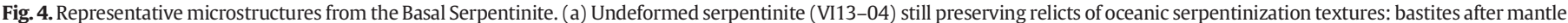

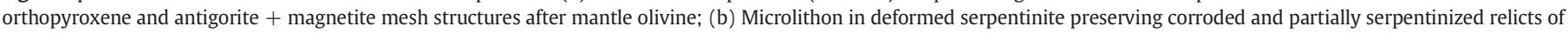

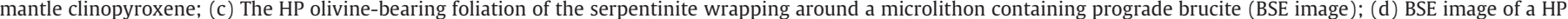

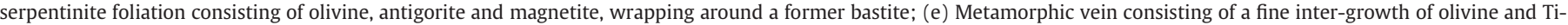

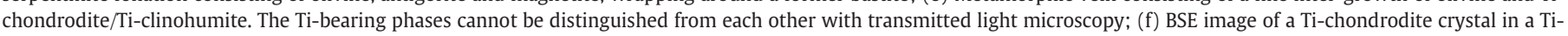

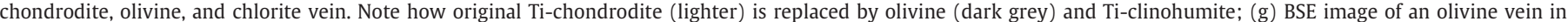
serpentinite. Note lighter olivine(1) and darker olivine(2), replacing original vein antigorite; (h) Eclogite from within the Basal Serpentinite. 
Table 2

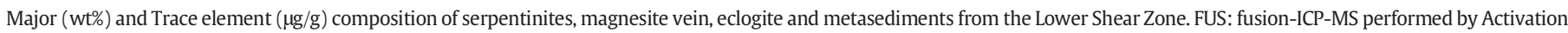

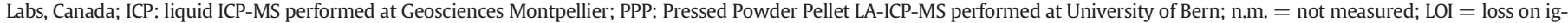
nition; b.d.l. = below detection limit.

\begin{tabular}{|c|c|c|c|c|c|c|c|c|c|c|c|c|}
\hline & \multicolumn{12}{|c|}{ Monviso lower shear zone } \\
\hline & \multicolumn{12}{|c|}{ Serpentinite } \\
\hline & \multicolumn{3}{|c|}{ GLSZ 1401} & \multicolumn{3}{|c|}{ GLSZ 1402} & \multicolumn{3}{|c|}{ GLSZ 1411} & \multicolumn{3}{|c|}{ GLSZ 1425} \\
\hline & FUS & ICP & PPP & FUS & ICP & PPP & FUS & ICP & PPP & FUS & ICP & PPP \\
\hline $\mathrm{SiO} 2$ & 40.42 & & 40.89 & 39.73 & & 40.36 & 40.97 & & 41.24 & 39.14 & & 39.75 \\
\hline $\mathrm{Al} 2 \mathrm{O} 3$ & 2.35 & & 2.21 & 2.03 & & 1.92 & 2.14 & & 2.03 & 2.87 & & 2.73 \\
\hline $\mathrm{Fe} 2 \mathrm{O} 3(\mathrm{~T})$ & 8.94 & & 8.87 & 8.56 & & 8.52 & 8.22 & & 8.07 & 9.23 & & 8.85 \\
\hline $\mathrm{MnO}$ & 0.11 & 0.11 & 0.1 & 0.11 & 0.11 & 0.1 & 0.07 & 0.065 & 0.06 & 0.1 & 0.091 & 0.09 \\
\hline $\mathrm{MgO}$ & 36.22 & & 36.27 & 36.64 & & 36.58 & 36.68 & & 36.85 & 36.27 & & 36.19 \\
\hline $\mathrm{CaO}$ & 0.02 & & 0.03 & 0.47 & & 0.51 & 0.04 & & 0.05 & 1.18 & & 1.17 \\
\hline $\mathrm{Na} 2 \mathrm{O}$ & $<0.01$ & & 0.003 & $<0.01$ & & 0.005 & $<0.01$ & & 0.01 & $<0.01$ & & 0.004 \\
\hline K2O & $<0.01$ & & 0.001 & $<0.01$ & & 0.001 & $<0.01$ & & 0.002 & $<0.01$ & & 0.002 \\
\hline $\mathrm{TiO} 2$ & 0.09 & 0.094 & 0.09 & 0.09 & 0.09 & 0.09 & 0.07 & 0.061 & 0.06 & 0.04 & 0.034 & 0.03 \\
\hline P2O5 & $<0.01$ & & 0.004 & $<0.01$ & & 0.007 & 0.01 & & 0.001 & $<0.01$ & & 0.001 \\
\hline LOI & 11.55 & & 11.55 & 11.91 & & 11.91 & 11.62 & & 11.62 & 11.17 & & 11.17 \\
\hline Total & 99.71 & & 100.01 & 99.54 & & 100.00 & 99.81 & & 100.00 & 99.99 & & 99.99 \\
\hline $\mathrm{Li}$ & & 0.267 & 0.226 & & 0.052 & 0.082 & & 0.159 & 0.108 & & 0.15 & 0.073 \\
\hline $\mathrm{Be}$ & $<1$ & n.m. & 0.082 & $<1$ & n.m. & 0.073 & $<1$ & n.m. & 0.087 & $<1$ & n.m. & 0.073 \\
\hline B & & n.m. & 7.21 & & n.m. & 7.76 & & n.m. & 14.4 & & n.m. & 11.7 \\
\hline Sc & 11 & 12 & 12.3 & 11 & 11.4 & 12.2 & 10 & 9.68 & 11.3 & 13 & 12.3 & 13.7 \\
\hline V & 66 & 70.7 & 66.2 & 55 & 57.2 & 53.7 & 49 & 44.6 & 46.6 & 71 & 66.2 & 67.3 \\
\hline $\mathrm{Cr}$ & & n.m. & 2270 & & n.m. & 2150 & & n.m. & 2600 & & n.m. & 2320 \\
\hline Co & & 85.7 & 98 & & 83.6 & 98.9 & & 96.6 & 102 & & 94.2 & 96.2 \\
\hline $\mathrm{Ni}$ & & 1710 & 1920 & & 1680 & 1950 & & 2010 & 2120 & & 1800 & 1840 \\
\hline $\mathrm{Cu}$ & & 5.22 & 6.26 & & 4.38 & 5.61 & & 2.88 & 4.58 & & 17.7 & 21 \\
\hline $\mathrm{Zn}$ & & 56.4 & 38.4 & & 46.7 & 29.1 & & 50.3 & 28 & & 43.5 & 23.5 \\
\hline $\mathrm{Ga}$ & & 2.74 & 3.1 & & 2.1 & 2.38 & & 2.44 & 2.63 & & 2.67 & 2.67 \\
\hline $\mathrm{Ge}$ & & n.m. & 0.73 & & n.m. & 0.764 & & n.m. & 0.549 & & n.m. & 0.874 \\
\hline As & & b.d.l. & 0.065 & & 0.621 & 0.464 & & b.d.l. & 0.107 & & b.d.l. & 0.211 \\
\hline $\mathrm{Se}$ & & n.m. & $<0.143$ & & n.m. & $<0.143$ & & n.m. & 0.127 & & n.m. & 0.136 \\
\hline $\mathrm{Rb}$ & & 0.057 & 0.05 & & 0.095 & 0.084 & & 0.053 & 0.049 & & 0.051 & 0.058 \\
\hline $\mathrm{Sr}$ & $<2$ & 0.28 & 0.368 & 7 & 5.27 & 5.77 & $<2$ & 0.402 & 0.54 & 2 & 0.899 & 0.926 \\
\hline $\mathrm{Y}$ & 3 & 1.65 & 1.67 & 3 & 1.36 & 1.48 & 2 & 0.484 & 0.518 & 2 & 1.56 & 1.63 \\
\hline $\mathrm{Zr}$ & 4 & 0.113 & 1.97 & 8 & 0.281 & 5.76 & 3 & 0.227 & 1.05 & 3 & 0.253 & 1.07 \\
\hline $\mathrm{Nb}$ & & 0.106 & 0.093 & & 0.443 & 0.373 & & 0.206 & 0.199 & & 0.023 & 0.032 \\
\hline Mo & & n.m. & 0.042 & & n.m. & 0.049 & & n.m. & 0.050 & & n.m. & 0.040 \\
\hline $\mathrm{Cd}$ & & n.m. & $<0.015$ & & n.m. & $<0.014$ & & n.m. & 0.016 & & n.m. & $<0.013$ \\
\hline In & & n.m. & 0.015 & & n.m. & 0.010 & & n.m. & 0.012 & & n.m. & 0.011 \\
\hline Sn & & 0.259 & 0.221 & & 0.329 & 0.266 & & 0.166 & 0.137 & & 0.153 & 0.149 \\
\hline $\mathrm{Sb}$ & & 0.005 & 0.007 & & 0.016 & 0.013 & & 0.017 & 0.014 & & 0.026 & 0.02 \\
\hline Cs & & 0.03 & 0.029 & & 0.092 & 0.083 & & 0.014 & 0.016 & & 0.009 & 0.011 \\
\hline $\mathrm{Ba}$ & $<2$ & 0.216 & 2.780 & $<2$ & 0.456 & 1.14 & $<2$ & 0.856 & 1.56 & 4 & 2.79 & 3.31 \\
\hline $\mathrm{La}$ & & 0.06 & 0.057 & & 0.069 & 0.069 & & 0.041 & 0.040 & & 0.085 & 0.085 \\
\hline $\mathrm{Ce}$ & & 0.207 & 0.206 & & 0.244 & 0.257 & & 0.101 & 0.109 & & 0.269 & 0.287 \\
\hline $\operatorname{Pr}$ & & 0.04 & 0.039 & & 0.046 & 0.048 & & 0.018 & 0.018 & & 0.048 & 0.049 \\
\hline $\mathrm{Nd}$ & & 0.237 & 0.237 & & 0.267 & 0.297 & & 0.091 & 0.102 & & 0.275 & 0.282 \\
\hline $\mathrm{Sm}$ & & 0.101 & 0.102 & & 0.097 & 0.113 & & 0.033 & 0.032 & & 0.112 & 0.112 \\
\hline $\mathrm{Eu}$ & & 0.026 & 0.026 & & 0.034 & 0.032 & & 0.010 & 0.010 & & 0.029 & 0.031 \\
\hline $\mathrm{Gd}$ & & 0.171 & 0.163 & & 0.156 & 0.176 & & 0.055 & 0.053 & & 0.197 & 0.197 \\
\hline $\mathrm{Tb}$ & & 0.035 & 0.033 & & 0.028 & 0.029 & & 0.010 & 0.010 & & 0.039 & 0.036 \\
\hline Dy & & 0.262 & 0.246 & & 0.206 & 0.202 & & 0.074 & 0.077 & & 0.275 & 0.282 \\
\hline Ho & & 0.063 & 0.063 & & 0.049 & 0.049 & & 0.019 & 0.020 & & 0.065 & 0.062 \\
\hline $\mathrm{Er}$ & & 0.204 & 0.216 & & 0.161 & 0.177 & & 0.059 & 0.070 & & 0.193 & 0.204 \\
\hline $\mathrm{Tm}$ & & 0.035 & 0.034 & & 0.027 & 0.027 & & 0.010 & 0.010 & & 0.032 & 0.031 \\
\hline $\mathrm{Yb}$ & & 0.27 & 0.273 & & 0.204 & 0.21 & & 0.086 & 0.097 & & 0.226 & 0.226 \\
\hline $\mathrm{Lu}$ & & 0.048 & 0.047 & & 0.038 & 0.041 & & 0.017 & 0.018 & & 0.036 & 0.036 \\
\hline $\mathrm{Hf}$ & & 0.008 & 0.083 & & 0.021 & 0.264 & & 0.006 & 0.032 & & 0.022 & 0.054 \\
\hline $\mathrm{Ta}$ & & b.d.l. & 0.009 & & 0.021 & 0.017 & & 0.009 & 0.012 & & 0.001 & 0.001 \\
\hline W & & 0.212 & 0.183 & & 0.2 & 0.171 & & b.d.l. & 0.086 & & b.d.l. & 0.040 \\
\hline $\mathrm{Tl}$ & & n.m. & 0.002 & & n.m. & 0.002 & & n.m. & $<0.001$ & & n.m. & 0.002 \\
\hline $\mathrm{Pb}$ & & 0.216 & 0.186 & & 0.427 & 0.468 & & 0.353 & 0.393 & & 1.31 & 1.45 \\
\hline $\mathrm{Bi}$ & & n.m. & 0.002 & & n.m. & 0.002 & & n.m. & 0.002 & & n.m. & 0.003 \\
\hline Th & & 0.04 & 0.042 & & 0.024 & 0.024 & & 0.027 & 0.026 & & 0.006 & 0.007 \\
\hline $\mathrm{U}$ & & 0.005 & 0.006 & & 0.007 & 0.008 & & 0.010 & 0.010 & & 0.005 & 0.006 \\
\hline
\end{tabular}




\begin{tabular}{|c|c|c|c|c|c|c|c|c|c|c|c|c|}
\hline & \multicolumn{12}{|c|}{ Monviso lower shear zone } \\
\hline & \multicolumn{3}{|c|}{ Eclogite } & \multicolumn{6}{|c|}{ Metasediment } & \multicolumn{3}{|c|}{ MgsVein } \\
\hline & \multicolumn{3}{|c|}{ GLSZ 1412A } & \multicolumn{3}{|c|}{ GLSZ 1413A } & \multicolumn{3}{|c|}{ GLSZ 1413B } & \multicolumn{3}{|l|}{ V2 } \\
\hline & FUS & ICP & PPP & FUS & ICP & PPP & FUS & ICP & PPP & FUS & ICP & PPP \\
\hline $\mathrm{SiO} 2$ & 47.72 & & 49.03 & 16.03 & & 17.8 & 57.8 & & 59.76 & 1.09 & & 1.95 \\
\hline $\mathrm{Al} 2 \mathrm{O} 3$ & 15.14 & & 14.71 & 3.3 & & 3.35 & 20.79 & & 20.51 & 0.08 & & 0.09 \\
\hline $\mathrm{Fe} 2 \mathrm{O} 3(\mathrm{~T})$ & 13.95 & & 13.71 & 1.88 & & 2.23 & 6.63 & & 6.53 & 9.98 & & 10.63 \\
\hline $\mathrm{MnO}$ & 0.2 & 0.21 & 0.17 & 0.1 & 0.11 & 0.097 & 0.14 & 0.15 & 0.11 & 0.782 & 0.91 & 0.71 \\
\hline $\mathrm{MgO}$ & 8.86 & & 8.54 & 0.73 & & 0.93 & 2.08 & & 2.05 & 34.69 & & 34.68 \\
\hline $\mathrm{CaO}$ & 6.85 & & 6.91 & 42.4 & & 40.75 & 0.34 & & 0.51 & 5.24 & & 5.32 \\
\hline $\mathrm{Na} 2 \mathrm{O}$ & 4.87 & & 4.71 & 0.14 & & 0.19 & 2.04 & & 1.98 & $<0.01$ & & 0.01 \\
\hline $\mathrm{K} 2 \mathrm{O}$ & 0.03 & & 0.04 & 0.25 & & 0.45 & 2.11 & & 1.98 & $<0.01$ & & 0.02 \\
\hline TiO2 & 1.29 & 1.2 & 1.22 & 0.15 & 0.13 & 0.15 & 0.99 & 0.93 & 0.93 & 0.004 & 0.0035 & 0.003 \\
\hline P2O5 & 0.05 & & 0.03 & 0.05 & & 0.0409 & 0.07 & & 0.0588 & 0.34 & & 0.28 \\
\hline LOI & 0.94 & & 0.94 & 34.02 & & 34.02 & 5.57 & & 5.57 & 46.31 & & 46.31 \\
\hline Total & 99.88 & & 100.01 & 99.04 & & 100.01 & 98.55 & & 99.99 & 98.52 & & 100.00 \\
\hline $\mathrm{Li}$ & & 35.9 & 35.6 & & 26.9 & 21 & & 104 & 86.1 & & 0.219 & 0.137 \\
\hline $\mathrm{Be}$ & $<1$ & n.m. & 0.796 & $<1$ & n.m. & 0.435 & 2 & n.m. & 1.8 & $<1$ & n.m. & $<0.046$ \\
\hline B & 109 & n.m. & 3.06 & 28 & n.m. & 12.8 & 164 & n.m. & 78.5 & & n.m. & 0.882 \\
\hline $\mathrm{Sc}$ & 34 & 34.2 & 34.6 & 4 & 4.57 & 5.14 & 20 & 19.6 & 19.5 & 2 & 2.08 & 2.01 \\
\hline V & 302 & 355 & 323 & 29 & 30.1 & 29.4 & 151 & 167 & 157 & 6 & 2.2 & 1.8 \\
\hline $\mathrm{Cr}$ & & n.m. & 254 & & n.m. & 27.3 & & n.m. & 138 & & n.m. & 45.4 \\
\hline Co & & 43.7 & 40.8 & & 7.69 & 7.95 & & 29.8 & 27.5 & & 59.5 & 55.6 \\
\hline $\mathrm{Ni}$ & & 82.2 & 77.3 & & 16.6 & 17.6 & & 70.7 & 65.4 & & 539 & 501 \\
\hline $\mathrm{Cu}$ & & 4.85 & 5.07 & & 17.6 & 22.2 & & 58.3 & 58.6 & & 11 & 13.8 \\
\hline $\mathrm{Zn}$ & & 78.2 & 66.9 & & 28.1 & 25.9 & & 92.2 & 49.9 & & 23.7 & 24.8 \\
\hline $\mathrm{Ga}$ & & 20.7 & 18.1 & & 4.92 & 4.83 & & 29.6 & 24.7 & & 0.331 & 0.397 \\
\hline $\mathrm{Ge}$ & & n.m. & 1.04 & & n.m. & 1.13 & & n.m. & 3.46 & & n.m. & 0.217 \\
\hline As & & b.d.l. & 0.063 & & 2.12 & 1.64 & & 12.5 & 7.51 & & b.d.l. & 0.195 \\
\hline $\mathrm{Se}$ & & n.m. & 0.372 & & n.m. & 0.566 & & n.m. & 0.333 & & n.m. & 0.738 \\
\hline $\mathrm{Rb}$ & & 0.514 & 0.575 & & 19.7 & 22.1 & & 99.6 & 90.7 & & 0.081 & 0.186 \\
\hline $\mathrm{Sr}$ & 37 & 37.5 & 38.4 & 652 & 693 & 707 & 310 & 318 & 285 & 105 & 123 & 120 \\
\hline $\mathrm{Y}$ & 22 & 29.2 & 26 & 17 & 16.3 & 17.9 & 29 & 29.6 & 27.4 & 5 & 6.45 & 6.53 \\
\hline $\mathrm{Zr}$ & 109 & 8.36 & 105 & 28 & 3.26 & 30.2 & 164 & 14.9 & 143 & 3 & 0.111 & 0.188 \\
\hline $\mathrm{Nb}$ & & 2.29 & 1.76 & & 3.19 & 3.08 & & 21 & 16.5 & & 0.044 & 0.037 \\
\hline Mo & & n.m. & 1.34 & & n.m. & 0.181 & & n.m. & 0.357 & & n.m. & 0.331 \\
\hline $\mathrm{Cd}$ & & n.m. & 0.029 & & n.m. & 0.054 & & n.m. & 0.042 & & n.m. & 0.014 \\
\hline In & & n.m. & 0.122 & & n.m. & 0.019 & & n.m. & 0.075 & & n.m. & 0.003 \\
\hline Sn & & 4.82 & 3.57 & & 0.487 & 0.394 & & 3.59 & 2.77 & & 0.018 & 0.014 \\
\hline $\mathrm{Sb}$ & & b.d.l. & 0.043 & & 0.090 & 0.100 & & 0.619 & 0.591 & & b.d.l. & 0.007 \\
\hline Cs & & 0.136 & 0.131 & & 0.995 & 1.18 & & 4.92 & 4.66 & & 0.009 & 0.014 \\
\hline $\mathrm{Ba}$ & 12 & 10.4 & 13.7 & 42 & 41.3 & 47.2 & 190 & 179 & 163 & 2 & 1.26 & 1.73 \\
\hline $\mathrm{La}$ & & 3.52 & 3.02 & & 11.5 & 12.8 & & 39.6 & 32.8 & & 0.935 & 0.938 \\
\hline $\mathrm{Ce}$ & & 10.1 & 9.32 & & 19.5 & 23.2 & & 82 & 76.8 & & 3.33 & 3.59 \\
\hline $\operatorname{Pr}$ & & 1.69 & 1.52 & & 2.72 & 3.04 & & 9.04 & 7.93 & & 0.568 & 0.564 \\
\hline $\mathrm{Nd}$ & & 8.92 & 8.37 & & 11.2 & 12.9 & & 35.1 & 31.4 & & 2.94 & 3.04 \\
\hline $\mathrm{Sm}$ & & 2.89 & 2.78 & & 2.54 & 2.93 & & 7 & 6.36 & & 0.784 & 0.792 \\
\hline $\mathrm{Eu}$ & & 1.4 & 1.38 & & 0.647 & 0.756 & & 1.56 & 1.42 & & 0.206 & 0.21 \\
\hline Gd & & 3.87 & 3.6 & & 2.92 & 2.89 & & 7.09 & 5.41 & & 1.11 & 1.08 \\
\hline $\mathrm{Tb}$ & & 0.662 & 0.599 & & 0.396 & 0.398 & & 0.937 & 0.771 & & 0.158 & 0.147 \\
\hline Dy & & 4.58 & 4.31 & & 2.33 & 2.5 & & 5.35 & 4.91 & & 0.98 & 0.984 \\
\hline Ho & & 1.01 & 0.943 & & 0.476 & 0.476 & & 1.02 & 0.973 & & 0.221 & 0.208 \\
\hline $\mathrm{Er}$ & & 2.93 & 2.9 & & 1.38 & 1.38 & & 2.81 & 2.81 & & 0.671 & 0.66 \\
\hline $\mathrm{Tm}$ & & 0.414 & 0.383 & & 0.199 & 0.187 & & 0.407 & 0.386 & & 0.099 & 0.092 \\
\hline $\mathrm{Yb}$ & & 2.71 & 2.59 & & 1.31 & 1.27 & & 2.6 & 2.58 & & 0.725 & 0.71 \\
\hline $\mathrm{Lu}$ & & 0.396 & 0.373 & & 0.186 & 0.174 & & 0.336 & 0.349 & & 0.149 & 0.14 \\
\hline Hf & & 0.292 & 2.38 & & 0.086 & 0.623 & & 0.533 & 3.8 & & 0.006 & 0.006 \\
\hline Ta & & 0.131 & 0.115 & & 0.154 & 0.15 & & 1.24 & 1.06 & & 0.002 & 0.002 \\
\hline W & & b.d.l. & 0.269 & & b.d.l. & 0.342 & & 2.46 & 1.98 & & b.d.l. & 0.047 \\
\hline $\mathrm{Tl}$ & & n.m. & 0.005 & & n.m. & 0.105 & & n.m. & 0.422 & & n.m. & 0.036 \\
\hline $\mathrm{Pb}$ & & 0.272 & 0.273 & & 12.6 & 14.5 & & 27.8 & 27.8 & & 0.349 & 0.493 \\
\hline $\mathrm{Bi}$ & & n.m. & 0.005 & & n.m. & 0.105 & & n.m. & 0.362 & & n.m. & 0.003 \\
\hline Th & & 0.109 & 0.105 & & 2.29 & 2.84 & & 14.4 & 13.1 & & 0.012 & 0.015 \\
\hline $\mathrm{U}$ & & 0.035 & 0.08 & & 0.261 & 0.515 & & 2.11 & 2.68 & & 0.004 & 0.007 \\
\hline
\end{tabular}

of oceanic basalts $\left(\mathrm{Al}_{2} \mathrm{O}_{3}=15.14 \mathrm{wt} \%, \mathrm{TiO}_{2}=1.29 \mathrm{wt} \%, \mathrm{CaO}=6.85 \mathrm{wt}\right.$ $\%, \mathrm{Na}_{2} \mathrm{O}=4.87 \mathrm{wt} \%$, and L.O.I. content of $0.9 \mathrm{wt} \%$ ).

The Basal Serpentinite displays a compositional variability like the LSZ serpentinite regarding $\mathrm{CaO}(0.2-1.5 \mathrm{wt} \%)$ and $\mathrm{Al}_{2} \mathrm{O}_{3}$ (1.2-2.3 wt\%; Fig. 5a). However, FeO (2.7-9.6 wt\%) and MgO (34.6-40.5 wt\%) contents vary more, as does the $\mathrm{Mg \#} \mathrm{(molar} \mathrm{Mg} /(\mathrm{Mg}+\mathrm{Fe})$; Fig. $5 \mathrm{~b})$. In general, the Basal Serpentinite displays higher $\mathrm{MgO}$ and lower $\mathrm{Al}_{2} \mathrm{O}_{3}$ contents (except for sample GLSZ-1410A; Tables 2-3 and Fig. 5c), reflecting different levels of depletion in the pristine mantle. This difference in $\mathrm{MgO}$ vs. $\mathrm{Al}_{2} \mathrm{O}_{3}$ in the bulk rock also influences the composition of the main rock forming minerals (see section 6.2.1) The Ti-chondrodite/clinohumite bulk veins from the Basal Serpentinite display variable major element compositions, especially for their $\mathrm{FeO}$ (9.7-11.7 wt\%) and $\mathrm{Al}_{2} \mathrm{O}_{3}(1.2-3.7 \mathrm{wt} \%)$ contents. This difference reflects their variable modal abundance of magnetite, diopside, olivine, and Ti-chondrodite/Ti-clinohumite.

The composition of the eclogite within the Basal Serpentinite (sample \#06) is comparable to the eclogite in the LSZ (sample \#12A) and has 
Table 3

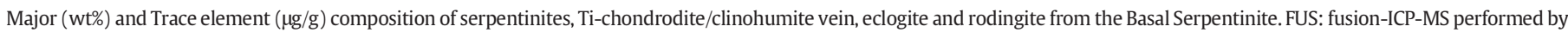

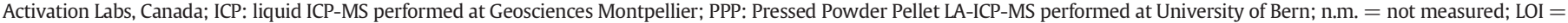
loss on ignition; b.d.l. = below detection limit.

\begin{tabular}{|c|c|c|c|c|c|c|c|c|c|c|c|c|c|c|c|}
\hline & \multicolumn{15}{|c|}{ Monviso sole thrust } \\
\hline & \multicolumn{15}{|c|}{ Serpentinite } \\
\hline & \multicolumn{3}{|c|}{ GLSZ 1409A } & \multicolumn{3}{|c|}{ GLSZ 1410A } & \multicolumn{3}{|c|}{ GLSZ 1415} & \multicolumn{3}{|c|}{ GLSZ 1417} & \multicolumn{3}{|c|}{ VI 1304} \\
\hline & FUS & ICP & PPP & FUS & ICP & PPP & FUS & ICP & PPP & FUS & ICP & PPP & FUS & ICP & PPP \\
\hline $\mathrm{SiO} 2$ & 39.85 & & 40.38 & 38.41 & & 38.82 & 41.88 & & 41.9 & 39.39 & & 39.92 & 39.71 & & 41.15 \\
\hline $\mathrm{Al} 2 \mathrm{O} 3$ & 1.69 & & 1.57 & 2.29 & & 2.22 & 1.7 & & 1.63 & 1.34 & & 1.29 & 1.56 & & 1.51 \\
\hline $\mathrm{Fe} 2 \mathrm{O} 3(\mathrm{~T})$ & 7.26 & & 7.15 & 10.69 & & 10.69 & 2.96 & & 2.99 & 6.11 & & 6.22 & 4.96 & & 5.09 \\
\hline $\mathrm{MnO}$ & 0.09 & 0.087 & 0.08 & 0.07 & 0.065 & 0.06 & 0.1 & 0.091 & 0.09 & 0.14 & 0.13 & 0.12 & 0.12 & 0.11 & 0.11 \\
\hline $\mathrm{MgO}$ & 38.89 & & 38.75 & 34.56 & & 35.58 & 40.42 & & 40.51 & 38.58 & & 39.6 & 38.51 & & 39.57 \\
\hline $\mathrm{CaO}$ & 0.25 & & 0.24 & 1.71 & & 1.76 & 0.04 & & 0.04 & 0.81 & & 0.83 & 0.03 & & 0.04 \\
\hline $\mathrm{Na} 2 \mathrm{O}$ & $<0.01$ & & 0.003 & $<0.01$ & & 0.008 & $<0.01$ & & 0.004 & 0.01 & & 0.01 & 0.01 & & 0.01 \\
\hline $\mathrm{K} 2 \mathrm{O}$ & $<0.01$ & & 0.001 & $<0.01$ & & 0.001 & $<0.01$ & & 0.002 & $<0.01$ & & 0.002 & $<0.01$ & & 0.0005 \\
\hline $\mathrm{TiO} 2$ & 0.02 & 0.016 & 0.02 & 0.07 & 0.071 & 0.07 & 0.02 & 0.015 & 0.02 & 0.03 & 0.025 & 0.03 & 0.025 & 0.026 & 0.02 \\
\hline P2O5 & $<0.01$ & & 0.001 & $<0.01$ & & 0.0008 & $<0.01$ & & 0.001 & $<0.01$ & & 0.002 & $<0.01$ & & 0.0007 \\
\hline LOI & 12.01 & & 12.01 & 10.88 & & 10.88 & 12.82 & & 12.82 & 11.98 & & 11.98 & 13.65 & & 13.65 \\
\hline Total & 100.10 & & 100.21 & 98.71 & & 100.10 & 99.94 & & 100.00 & 98.38 & & 100.00 & 98.58 & & 101.16 \\
\hline $\mathrm{Li}$ & & b.d.l. & 0.058 & & 0.065 & 0.185 & & 0.147 & 0.060 & & 0.727 & 0.652 & & 0.077 & 0.097 \\
\hline $\mathrm{Be}$ & $<1$ & n.m. & $<0.05$ & $<1$ & n.m. & 0.096 & $<1$ & n.m. & $<0.04$ & $<1$ & n.m. & 0.059 & $<1$ & n.m. & $<0.06$ \\
\hline B & & n.m. & 7.3 & & n.m. & 5.71 & & n.m. & 11.5 & & n.m. & 11.1 & & n.m. & 12.2 \\
\hline $\mathrm{Sc}$ & 10 & 9.72 & 10.6 & 15 & 15.1 & 16.3 & 8 & 8.06 & 9.07 & 12 & 11.5 & 13.3 & 9 & 9.66 & 10.4 \\
\hline V & 50 & 51.2 & 49.9 & 80 & 84.2 & 80.3 & 32 & 31.2 & 31.3 & 42 & 41.4 & 41.9 & 39 & 41.2 & 38.9 \\
\hline $\mathrm{Cr}$ & & n.m. & 2360 & & n.m. & 2700 & & n.m. & 2080 & & n.m. & 2140 & & n.m. & 2310 \\
\hline Co & & 85.3 & 100 & & 72.2 & 86.1 & & 80.2 & 84.3 & & 88.1 & 91.6 & & 84.1 & 99.7 \\
\hline $\mathrm{Ni}$ & & 1670 & 1930 & & 1440 & 1610 & & 1760 & 1860 & & 1630 & 1680 & & 1600 & 1870 \\
\hline $\mathrm{Cu}$ & & 12.2 & 14.6 & & 19.5 & 20.4 & & 2.48 & 4.17 & & 3.43 & 5.5 & & 28.1 & 34.4 \\
\hline $\mathrm{Zn}$ & & 40.7 & 22 & & 44.6 & 39.2 & & 38.6 & 14.8 & & 50.4 & 27.1 & & 53.2 & 32.3 \\
\hline $\mathrm{Ga}$ & & 1.42 & 1.64 & & 2.17 & 2.48 & & 1.49 & 1.58 & & 1.53 & 1.58 & & 1.58 & 1.81 \\
\hline $\mathrm{Ge}$ & & n.m. & 0.652 & & n.m. & 0.766 & & n.m. & 0.682 & & n.m. & 0.726 & & n.m. & 0.738 \\
\hline As & & b.d.l. & 0.048 & & b.d.l. & 0.107 & & b.d.l. & 0.215 & & 1.6 & 0.781 & & b.d.l. & 0.073 \\
\hline $\mathrm{Se}$ & & n.m. & $<0.12$ & & n.m. & $<0.129$ & & n.m. & $<0.128$ & & n.m. & $<0.13$ & & n.m. & $<0.113$ \\
\hline $\mathrm{Rb}$ & & 0.012 & 0.018 & & 0.017 & 0.024 & & 0.059 & 0.061 & & 0.037 & 0.046 & & 0.024 & 0.031 \\
\hline $\mathrm{Sr}$ & $<2$ & 0.212 & 0.34 & 3 & 1.87 & 2.18 & $<2$ & 0.772 & 0.842 & 17 & 15.7 & 15.6 & $<2$ & 0.628 & 0.834 \\
\hline Y & 3 & 0.555 & 0.569 & 2 & 1.67 & 1.71 & 5 & 0.892 & 0.937 & 4 & 1.45 & 1.22 & 4 & 1.22 & 1.3 \\
\hline $\mathrm{Zr}$ & 3 & 0.171 & 0.349 & 6 & 4.36 & 4.55 & 3 & 0.541 & 0.505 & 5 & 3.57 & 4.29 & 3 & 1.33 & 1.86 \\
\hline $\mathrm{Nb}$ & & 0.012 & 0.014 & & 0.31 & 0.268 & & 0.090 & 0.084 & & 0.093 & 0.088 & & 0.037 & 0.034 \\
\hline Mo & & n.m. & 0.066 & & n.m. & 0.051 & & n.m. & 0.057 & & n.m. & 0.072 & & n.m. & 0.083 \\
\hline $\mathrm{Cd}$ & & n.m. & $<0.013$ & & n.m. & $<0.013$ & & n.m. & 0.023 & & n.m. & 0.015 & & n.m. & 0.011 \\
\hline In & & n.m. & 0.006 & & n.m. & 0.011 & & n.m. & 0.004 & & n.m. & 0.009 & & n.m. & 0.008 \\
\hline Sn & & 0.038 & 0.027 & & 0.333 & 0.266 & & 0.0479 & 0.039 & & 0.082 & 0.068 & & 0.16 & 0.089 \\
\hline $\mathrm{Sb}$ & & 0.004 & 0.007 & & 0.036 & 0.032 & & 0.283 & 0.212 & & 0.128 & 0.075 & & 0.028 & 0.022 \\
\hline Cs & & 0.001 & 0.003 & & 0.002 & 0.006 & & 0.002 & $<0.002$ & & 0.008 & 0.001 & & 0.003 & 0.003 \\
\hline $\mathrm{Ba}$ & $<2$ & 0.369 & 1.1 & $<2$ & 0.364 & 1.87 & $<2$ & 0.586 & 0.984 & 3 & 2.18 & 2.64 & $<2$ & 0.212 & 1.69 \\
\hline La & & 0.066 & 0.063 & & 0.33 & 0.318 & & 0.117 & 0.104 & & 0.159 & 0.148 & & 0.11 & 0.113 \\
\hline $\mathrm{Ce}$ & & 0.158 & 0.165 & & 0.964 & 0.973 & & 0.244 & 0.245 & & 0.543 & 0.519 & & 0.345 & 0.367 \\
\hline $\operatorname{Pr}$ & & 0.022 & 0.023 & & 0.155 & 0.154 & & 0.031 & 0.031 & & 0.101 & 0.093 & & 0.057 & 0.058 \\
\hline $\mathrm{Nd}$ & & 0.094 & 0.098 & & 0.762 & 0.768 & & 0.136 & 0.148 & & 0.535 & 0.5 & & 0.305 & 0.33 \\
\hline $\mathrm{Sm}$ & & 0.028 & 0.03 & & 0.211 & 0.222 & & 0.043 & 0.05 & & 0.164 & 0.153 & & 0.103 & 0.114 \\
\hline $\mathrm{Eu}$ & & 0.006 & 0.006 & & 0.038 & 0.036 & & 0.017 & 0.016 & & 0.0131 & 0.012 & & 0.02 & 0.021 \\
\hline Gd & & 0.049 & 0.055 & & 0.258 & 0.260 & & 0.079 & 0.071 & & 0.223 & 0.191 & & 0.153 & 0.165 \\
\hline $\mathrm{Tb}$ & & 0.010 & 0.010 & & 0.045 & 0.043 & & 0.015 & 0.015 & & 0.038 & 0.028 & & 0.028 & 0.029 \\
\hline Dy & & 0.082 & 0.072 & & 0.296 & 0.289 & & 0.127 & 0.125 & & 0.242 & 0.188 & & 0.202 & 0.204 \\
\hline Ho & & 0.021 & 0.021 & & 0.064 & 0.059 & & 0.032 & 0.03 & & 0.052 & 0.037 & & 0.046 & 0.048 \\
\hline $\mathrm{Er}$ & & 0.074 & 0.069 & & 0.181 & 0.181 & & 0.104 & 0.11 & & 0.155 & 0.113 & & 0.141 & 0.156 \\
\hline $\mathrm{Tm}$ & & 0.012 & 0.012 & & 0.031 & 0.031 & & 0.0165 & 0.017 & & 0.027 & 0.021 & & 0.023 & 0.025 \\
\hline $\mathrm{Yb}$ & & 0.095 & 0.095 & & 0.275 & 0.267 & & 0.109 & 0.114 & & 0.264 & 0.223 & & 0.164 & 0.172 \\
\hline $\mathrm{Lu}$ & & 0.017 & 0.017 & & 0.057 & 0.055 & & 0.017 & 0.018 & & 0.062 & 0.053 & & 0.027 & 0.027 \\
\hline $\mathrm{Hf}$ & & b.d.l. & 0.011 & & 0.169 & 0.158 & & 0.015 & 0.016 & & 0.183 & 0.191 & & 0.054 & 0.06 \\
\hline Тa & & 0.001 & $<0.001$ & & 0.019 & 0.013 & & 0.003 & 0.005 & & 0.005 & 0.007 & & 0.0023 & 0.001 \\
\hline W & & 0.047 & 0.043 & & 0.181 & 0.167 & & b.d.l. & 0.237 & & b.d.l. & 0.36 & & 0.952 & 0.949 \\
\hline $\mathrm{Tl}$ & & n.m. & 0.001 & & n.m. & 0.001 & & n.m. & 0.001 & & n.m. & 0.001 & & n.m. & 0.002 \\
\hline $\mathrm{Pb}$ & & 0.562 & 0.592 & & 0.362 & 0.445 & & 0.28 & 0.297 & & 0.182 & 0.202 & & 0.648 & 0.329 \\
\hline $\mathrm{Bi}$ & & n.m. & 0.002 & & n.m. & $<0.001$ & & n.m. & 0.003 & & n.m. & 0.002 & & n.m. & 0.002 \\
\hline Th & & 0.002 & 0.003 & & 0.042 & 0.043 & & 0.016 & 0.015 & & 0.038 & 0.035 & & 0.007 & 0.007 \\
\hline $\mathrm{U}$ & & 0.0011 & 0.003 & & 0.009 & 0.01 & & 0.005 & 0.006 & & 0.008 & 0.008 & & 0.003 & 0.004 \\
\hline
\end{tabular}




\begin{tabular}{|c|c|c|c|c|c|c|c|c|c|c|c|c|}
\hline & \multicolumn{12}{|c|}{ Monviso sole thrust } \\
\hline & \multirow{2}{*}{\multicolumn{3}{|c|}{$\frac{\text { Olivine vein }}{\text { GLSZ 1410B }}$}} & \multicolumn{6}{|c|}{ Ti-Clinohuminte Vein } & \multirow{2}{*}{\multicolumn{3}{|c|}{$\frac{\text { Eclogite }}{\text { GLSZ } 1406}$}} \\
\hline & & & & \multicolumn{3}{|c|}{ GLSZ 1416} & \multicolumn{3}{|c|}{ GLSZ 1418} & & & \\
\hline & FUS & $\mathrm{ICP}$ & PPP & FUS & $\mathrm{ICP}$ & PPP & FUS & ICP & PPP & FUS & ICP & PPP \\
\hline $\mathrm{SiO} 2$ & 37.63 & & 38.49 & 34.52 & & 36.17 & 35.69 & & 36.43 & 50.48 & & 52.37 \\
\hline $\mathrm{Al} 2 \mathrm{O} 3$ & 1.17 & & 1.11 & 3.68 & & 3.32 & 1.18 & & 1.09 & 12.57 & & 11.63 \\
\hline $\mathrm{Fe} 2 \mathrm{O} 3(\mathrm{~T})$ & 10.56 & & 10.14 & 9.68 & & 9.9 & 11.72 & & 11.38 & 14.01 & & 13.34 \\
\hline $\mathrm{MnO}$ & 0.18 & 0.16 & 0.15 & 0.17 & 0.14 & 0.15 & 0.3 & 0.41 & 0.26 & 0.22 & 0.21 & 0.18 \\
\hline $\mathrm{MgO}$ & 40.27 & & 40.11 & 41.69 & & 41.57 & 42.65 & & 42.61 & 6.16 & & 6 \\
\hline $\mathrm{CaO}$ & 0.64 & & 0.65 & 0.42 & & 0.45 & 1.73 & & 1.83 & 9.71 & & 9.4 \\
\hline $\mathrm{Na} 2 \mathrm{O}$ & $<0.01$ & & 0.003 & 0.02 & & 0.08 & 0.02 & & 0.017 & 6.32 & & 5.91 \\
\hline K2O & $<0.01$ & & 0.0009 & $<0.01$ & & 0.01 & $<0.01$ & & 0.009 & $<0.01$ & & 0.02 \\
\hline $\mathrm{TiO} 2$ & 0.04 & 0.029 & 0.03 & 2.46 & 2.22 & 2.09 & 3.01 & 2.02 & 2.55 & 1.25 & 1.03 & 1.12 \\
\hline P2O5 & 0.01 & & 0.001 & 0.12 & & 0.09 & $<0.01$ & & 0.006 & 0.03 & & 0.02 \\
\hline LOI & 9.31 & & 9.31 & 6.17 & & 6.17 & 3.81 & & 3.81 & 0 & & 0 \\
\hline Total & 99.81 & & 100.00 & 98.92 & & 100.00 & 100.10 & & 99.99 & 100.74 & & 100.00 \\
\hline $\mathrm{Li}$ & & 0.268 & 0.28 & & 2.83 & 3.1 & & 4.92 & 5.1 & & 19.9 & 20.5 \\
\hline $\mathrm{Be}$ & $<1$ & n.m. & $<0.05$ & $<1$ & n.m. & 0.06 & $<1$ & n.m. & $<0.05$ & 1 & n.m. & 1.24 \\
\hline B & & n.m. & 10.8 & & n.m. & 13.1 & & n.m. & 22.7 & 1271 & n.m. & 2.21 \\
\hline Sc & 8 & 7.09 & 9.08 & 38 & 33.9 & 36.1 & 95 & 85.4 & 97.3 & 27 & 25.3 & 27.5 \\
\hline V & 36 & 35.9 & 34.2 & 153 & 139 & 152 & 138 & 83.1 & 131 & 31 & 25.3 & 25.7 \\
\hline $\mathrm{Cr}$ & & n.m. & 1790 & & n.m. & 156 & & n.m. & 523 & & n.m. & 9.23 \\
\hline $\mathrm{Co}$ & & 130 & 126 & & 109 & 118 & & 123 & 128 & & 24.4 & 24.3 \\
\hline $\mathrm{Ni}$ & & 2100 & 1960 & & 1540 & 1430 & & 1810 & 1880 & & 89.8 & 89.8 \\
\hline $\mathrm{Cu}$ & & 8.17 & 9.4 & & 6.91 & 6.02 & & 15.5 & 13.7 & & 2.08 & 2.92 \\
\hline Zn & & 49.4 & 31.7 & & 77.7 & 44.9 & & 68.9 & 52.6 & & 77.9 & 63.2 \\
\hline Ga & & 1.17 & 1.13 & & 3.82 & 4.33 & & 1.1 & 0.948 & & 31.2 & 27.8 \\
\hline Ge & & n.m. & 0.578 & & n.m. & 1.11 & & n.m. & 0.718 & & n.m. & 2.82 \\
\hline As & & 2.01 & 1.45 & & b.d.l. & 0.036 & & 0.372 & 0.012 & & b.d.l. & 0.15 \\
\hline $\mathrm{Se}$ & & n.m. & $<0.13$ & & n.m. & 0.243 & & n.m. & $<0.139$ & & n.m. & 0.688 \\
\hline $\mathrm{Rb}$ & & 0.011 & 0.016 & & 0.207 & 0.281 & & 0.184 & 0.197 & & 0.375 & 0.686 \\
\hline $\mathrm{Sr}$ & 6 & 4.31 & 4.59 & 11 & 8.86 & 9.13 & 25 & 21.3 & 22.6 & 41 & 36.4 & 38.2 \\
\hline Y & 1 & 0.577 & 0.661 & 6 & 6.49 & 6.51 & 2 & 3.49 & 3.85 & 94 & 99.5 & 93.2 \\
\hline $\mathrm{Zr}$ & 2 & 0.524 & 0.606 & 47 & 44 & 49.1 & 110 & 72.7 & 101 & 1271 & 127 & 993 \\
\hline $\mathrm{Nb}$ & & 0.005 & 0.011 & & 3.28 & 3.19 & & 2.4 & 3.17 & & 3.51 & 2.9 \\
\hline Mo & & n.m. & 0.132 & & n.m. & 0.111 & & n.m. & 0.14 & & n.m. & 0.198 \\
\hline $\mathrm{Cd}$ & & n.m. & $<0.107$ & & n.m. & 0.018 & & n.m. & $<0.016$ & & n.m. & 0.047 \\
\hline In & & n.m. & 0.005 & & n.m. & 0.143 & & n.m. & 0.053 & & n.m. & 0.153 \\
\hline Sn & & 0.113 & 0.032 & & 0.838 & 1.38 & & 0.344 & 0.171 & & 2.44 & 1.86 \\
\hline $\mathrm{Sb}$ & & 0.048 & 0.050 & & b.d.l. & 0.011 & & 0.181 & 0.017 & & b.d.l. & 0.029 \\
\hline Cs & & 0.002 & 0.002 & & 0.01 & 0.015 & & 0.011 & 0.014 & & 0.036 & 0.047 \\
\hline Ва & $<2$ & 0.549 & 1.19 & 8 & 6.35 & 8.19 & 6 & 5.22 & 5.67 & 10 & 8.63 & 9.62 \\
\hline $\mathrm{La}$ & & 0.018 & 0.017 & & 0.509 & 0.535 & & 0.423 & 0.4 & & 13.3 & 12.2 \\
\hline $\mathrm{Ce}$ & & 0.044 & 0.045 & & 1.67 & 2.05 & & 0.822 & 0.857 & & 41.5 & 41.6 \\
\hline $\mathrm{Pr}$ & & 0.007 & 0.006 & & 0.3 & 0.332 & & 0.099 & 0.098 & & 7.27 & 7.02 \\
\hline $\mathrm{Nd}$ & & 0.037 & 0.037 & & 1.65 & 1.81 & & 0.386 & 0.409 & & 38.8 & 38.4 \\
\hline $\mathrm{Sm}$ & & 0.019 & 0.018 & & 0.513 & 0.567 & & 0.108 & 0.123 & & 11.3 & 11.5 \\
\hline $\mathrm{Eu}$ & & 0.010 & 0.010 & & 0.114 & 0.128 & & 1.09 & 1.07 & & 3.43 & 3.56 \\
\hline Gd & & 0.049 & 0.045 & & 0.732 & 0.729 & & 0.182 & 0.191 & & 14.6 & 13.6 \\
\hline $\mathrm{Tb}$ & & 0.011 & 0.009 & & 0.122 & 0.121 & & 0.039 & 0.039 & & 2.45 & 2.23 \\
\hline Dy & & 0.091 & 0.095 & & 0.875 & 0.873 & & 0.357 & 0.351 & & 16.1 & 15.6 \\
\hline Ho & & 0.024 & 0.023 & & 0.236 & 0.221 & & 0.116 & & & 3.5 & 3.4 \\
\hline $\mathrm{Er}$ & & 0.085 & 0.078 & & 1.04 & 1.04 & & 0.62 & 0.649 & & 10.3 & 10.6 \\
\hline $\mathrm{Tm}$ & & 0.015 & 0.014 & & 0.303 & 0.277 & & 0.185 & 0.176 & & 1.46 & 1.45 \\
\hline $\mathrm{Yb}$ & & 0.112 & 0.102 & & 3.35 & 3.22 & & 2.18 & 2.13 & & 9.55 & 10.2 \\
\hline $\mathrm{Lu}$ & & 0.019 & 0.019 & & 0.664 & 0.632 & & 0.501 & 0.487 & & 1.44 & 1.55 \\
\hline $\mathrm{Hf}$ & & 0.022 & 0.022 & & 1.21 & 1.22 & & 1.73 & 1.97 & & 1.95 & 23.9 \\
\hline Та & & 0.0004 & $<0.001$ & & 0.241 & 0.209 & & 0.196 & 0.228 & & 0.236 & 0.22 \\
\hline W & & b.d.l. & 0.035 & & b.d.l. & 0.064 & & 1.37 & 1.59 & & 0.758 & 0.132 \\
\hline $\mathrm{Tl}$ & & n.m. & 0.001 & & n.m. & 0.002 & & n.m. & 0.002 & & n.m. & 0.006 \\
\hline $\mathrm{Pb}$ & & 0.229 & 0.193 & & 0.545 & 0.56 & & 8.8 & 7.55 & & 1.09 & 1.11 \\
\hline $\mathrm{Bi}$ & & n.m. & 0.001 & & n.m. & 0.001 & & n.m. & 0.001 & & n.m. & $<0.003$ \\
\hline Th & & 0.001 & $<0.002$ & & 0.053 & 0.046 & & 0.049 & 0.042 & & 0.384 & 0.409 \\
\hline U & & 0.0004 & 0.0024 & & 0.024 & 0.029 & & 0.021 & 0.021 & & 0.05 & 0.158 \\
\hline
\end{tabular}

$12.6 \mathrm{wt} \% \mathrm{Al}_{2} \mathrm{O}_{3}, 1.5 \mathrm{wt} \% \mathrm{TiO}_{2}, 9.71 \mathrm{wt} \% \mathrm{CaO}, 6.32 \mathrm{wt} \% \mathrm{Na}_{2} \mathrm{O}$, and L.O.I. content below detection.

\subsubsection{Trace elements}

Trace element compositions of the Monviso serpentinites can be compared with those of fresh oceanic mantle peridotite from Lanzo (Guarnieri et al., 2012), which consists of both depleted spinel harzburgite and of refertilized impregnated plagioclase peridotite. These Lanzo peridotites escaped significant oceanic serpentinization and thus largely preserved their unaltered mantle composition through the Alpine subduction cycle, thus providing useful information on the composition of the oceanic lithospheric mantle.

The REE patterns of two out of three LSZ serpentinites are comparable with those of fresh spinel harzburgites from the Alpine Lanzo peridotite (blue-shaded area in Fig. 6a; Guarnieri et al., 2012), whose REE contents are between 0.01 and 3 C1-chondrite. The REE patterns of the Basal Serpentinite (Fig. 6b) are comparable to those of the LSZ serpentinite for their LRRE, while their HRRE composition is slightly 

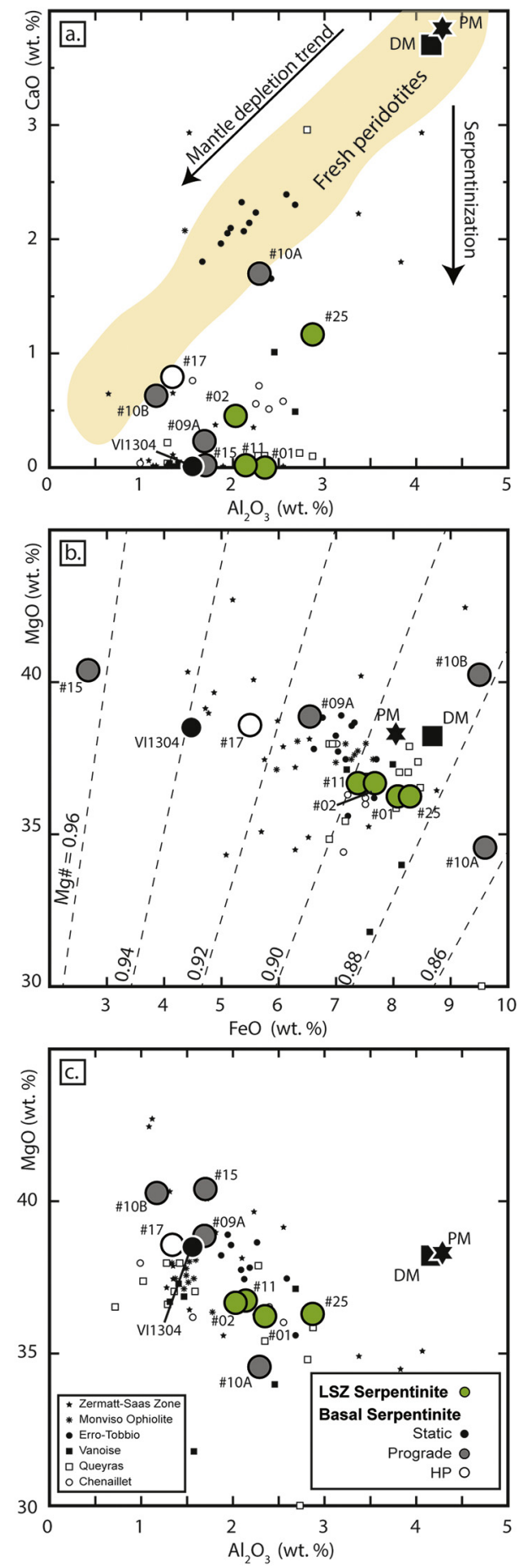

Fig. 5. Major element plots (a. $\mathrm{Al}_{2} \mathrm{O}_{3}$ vs $\mathrm{CaO}$; b. $\mathrm{FeO}$ vs $\mathrm{MgO}$; c. $\mathrm{Al}_{2} \mathrm{O}_{3}$ vs $\mathrm{MgO}$ ) for the Lower Shear Zone and for the Basal Serpentinite and veins. The shaded area refers to the melt depletion trend in peridotite. Literature data of serpentinites from Zermatt-Saas Zone ( $\mathrm{Li}$ et al., 2004), Monviso (Hattori and Guillot, 2007; Lafay et al., 2013), Erro-Tobbio (Scambelluri et al., 2001), Vanoise (Chalot-Prat et al., 2003), Queyras (Chalot-Prat et al., 2003; Hattori and Guillot, 2007; Lafay et al., 2013) and Chenaillet (Chalot-Prat et al., 2003; Hattori and Guillot, 2007; Lafay et al., 2013) are plotted for comparison. PM = primitive mantle and $\mathrm{DM}=$ depleted mantle.

more variable. Nevertheless, this indicates a strong similarity of mantle protolith producing the LSZ and Basal Serpentinite. These rocks also display comparable trace element compositions (Fig. 7). The LSZ serpentinite is prominently enriched by up to two orders of magnitude in $\mathrm{Cs}, \mathrm{B}, \mathrm{W}, \mathrm{K}, \mathrm{Pb}, \mathrm{As}$, and $\mathrm{Sb}$ with respect to the reference pristine Lanzo peridotite (Guarnieri et al., 2012). The Basal Serpentinite shows

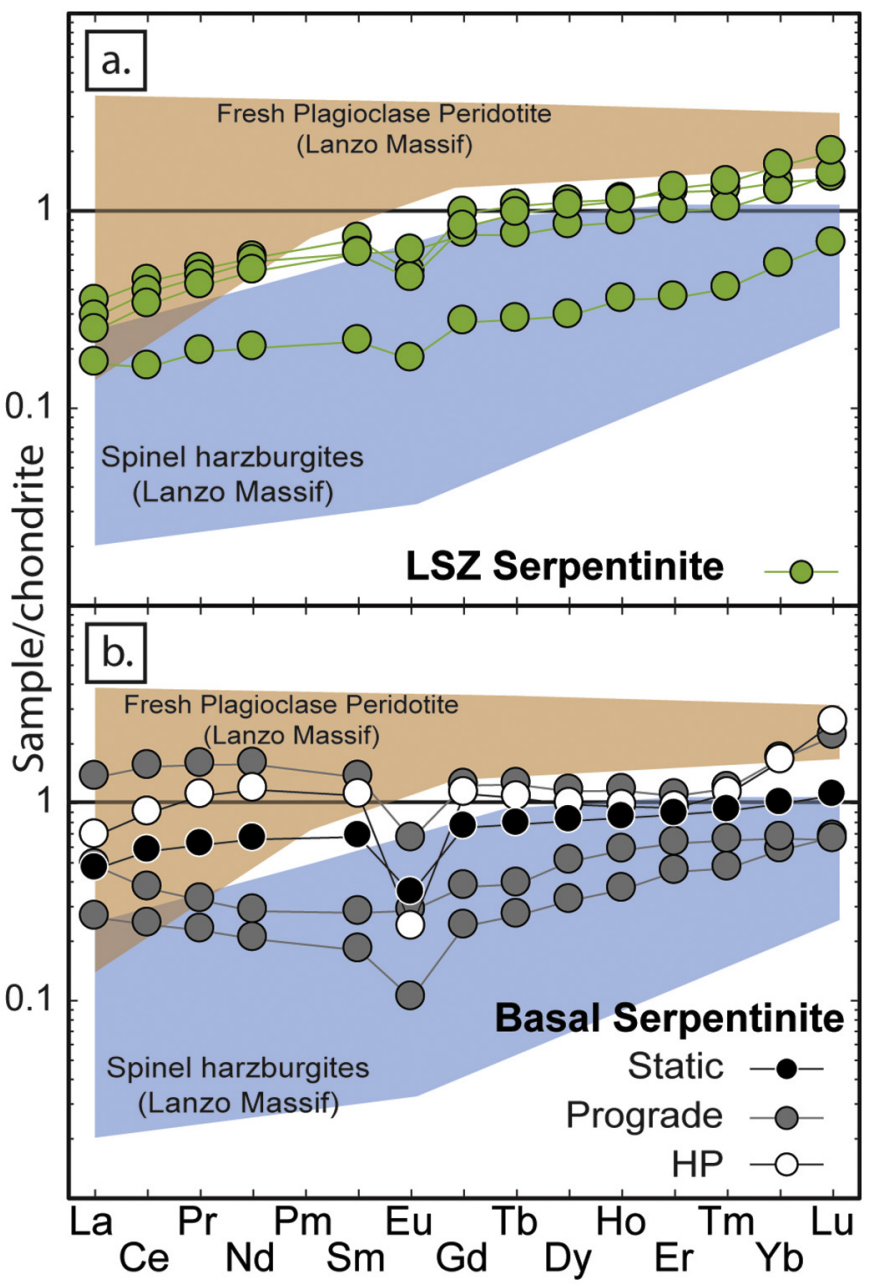

Fig. 6. C1 Chondrite-normalized (McDonough and Sun, 1995) REE patterns of bulk-rock from the Lower Shear Zone and Basal Serpentinite. Shaded areas are based on data for non-hydrated plagioclase peridotites and spinel harzburgites from Guarnieri et al. (2012).

enrichment in the same exact set of elements. Amongst such elements, $\mathrm{As}, \mathrm{Sb}$, and $\mathrm{Sr}$ show the highest variation within the Basal Serpentinite samples. In general, these elements are more abundant in the HP olivine-bearing Basal Serpentinite than in the static and the prograde ones.

\subsubsection{Sr and $\mathrm{Pb}$ isotopic compositions}

Selected samples of serpentinite and of olivine and Ti-clinohumite veins from the Lower Shear Zone and the Basal Serpentinite were analyzed for their $\mathrm{Pb}$ and $\mathrm{Sr}$ isotopic compositions. Results are shown in Table 4 and are displayed in Fig. 8.

The Lower Shear Zone serpentinites show ${ }^{87} \mathrm{Sr} /{ }^{86} \mathrm{Sr}$ ranging between 0.707131 and 0.709344 , i.e., values similar to and slightly higher than Jurassic seawater (Jones and Jenkyns, 2001). The Pb isotopic compositions of these serpentinites are similar throughout the entire LSZ, with ${ }^{206} \mathrm{~Pb} /{ }^{204} \mathrm{~Pb},{ }^{207} \mathrm{~Pb} /{ }^{204} \mathrm{~Pb}$, and ${ }^{208} \mathrm{~Pb} /{ }^{204} \mathrm{~Pb}$ values of $18.05-18.16$, 15.59-15.62 and 37.99-38.14, respectively.

The isotopic compositions of the Basal Serpentinite and of the Ticlinohumite veins are more variable when compared to those of the LSZ. The static (Sample VI13-04) and the HP serpentinite (\#17) have ${ }^{87} \mathrm{Sr} /{ }^{86} \mathrm{Sr}$ values of 0.706840 and 0.707362 , respectively, that are close to Jurassic seawater. The ${ }^{206} \mathrm{~Pb} /{ }^{204} \mathrm{~Pb},{ }^{207} \mathrm{~Pb} /{ }^{204} \mathrm{~Pb}$ and ${ }^{208} \mathrm{~Pb} /{ }^{204} \mathrm{~Pb}$ values of such samples (17.92, 15.47 and 37.67 respectively) are comparable with depleted mantle (DM) values. The olivine-bearing serpentinite (\#17) has ${ }^{206} \mathrm{~Pb} /{ }^{204} \mathrm{~Pb},{ }^{207} \mathrm{~Pb} /{ }^{204} \mathrm{~Pb}$ and ${ }^{208} \mathrm{~Pb} /{ }^{204} \mathrm{~Pb}$ values of $18.689,15.636$ and 38.651 respectively. The olivine vein (\#10B) has 


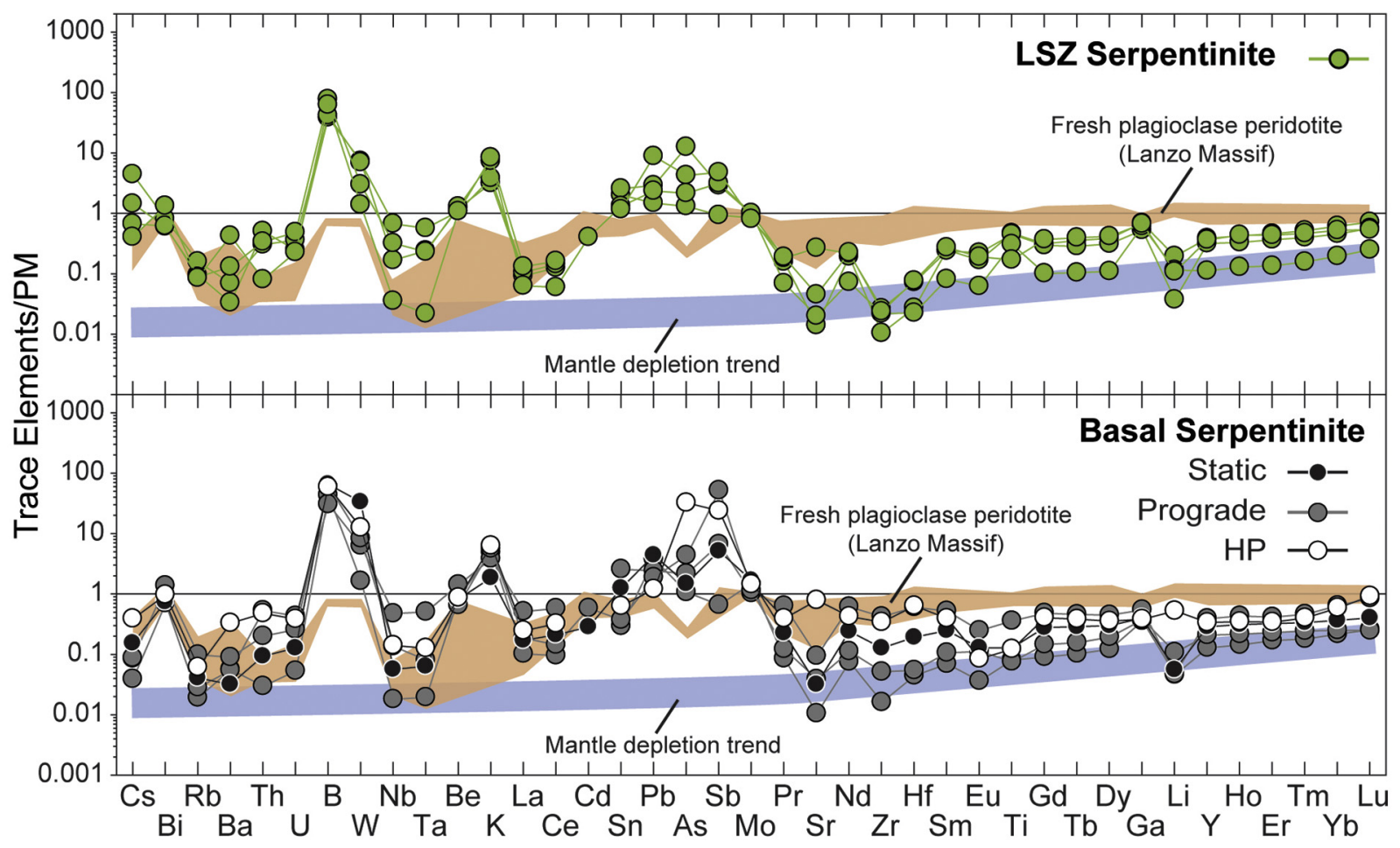

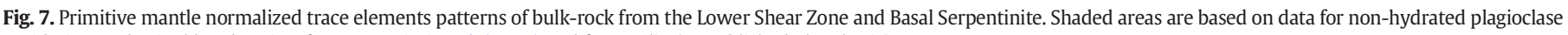
peridotites and spinel harzburgites from Guarnieri et al. (2012) and from Gilio (unpublished PhD thesis).

${ }^{87} \mathrm{Sr} /{ }^{86} \mathrm{Sr}=0.704891$, and ${ }^{206} \mathrm{~Pb} /{ }^{204} \mathrm{~Pb},{ }^{207} \mathrm{~Pb} /{ }^{204} \mathrm{~Pb}$ and ${ }^{208} \mathrm{~Pb} /{ }^{204} \mathrm{~Pb}$ values 18.218, 15.534 and 37.968 respectively. As shown in Fig. 8, one serpentinite sample and one Ti-clinohumite-bearing HP vein from the Basal Serpentinite display $\mathrm{Pb}$ isotope compositions overlapping the continental crust.

\subsection{Mineral compositions}

\subsubsection{Major elements}

Representative electron probe measurements of the main rockforming minerals of the Lower Shear Zone serpentinite, the magnesite vein, the Basal Serpentinite, and the olivine and Ti-clinohumite veins are reported in Tables 5-6. The full dataset is shown in the repository files.

In the LSZ serpentinites, $\operatorname{Atg}(1)^{\mathrm{LSZ}}$ has slightly higher $\mathrm{Al}$ content than $\operatorname{Atg}(2)^{\mathrm{LSZ}}, \sim 0.14-0.17$ and $\sim 0.10-0.12$ a.p.f.u., respectively (Fig. 9a). Mg\# in $\operatorname{Atg}(1)^{\mathrm{LSZ}}$ and $\operatorname{Atg}(2)^{\mathrm{LSZ}}$ vary between 0.89 and 0.94 , reflecting difference in bulk $\mathrm{FeO}$ and $\mathrm{MgO}$ content of the analyzed sample (\#01, \#11 and \#25). The carbonate veins (Fig. 2c-e) hosted in serpentinites consists of talc (Mg\# 95\%) and primary magnesite (Mgs 85-90\% and Sd $\sim 7-14 \%)$; the secondary dolomite has a composition of magnesite $\sim 47 \%$ and siderite $\sim 5 \%$. Late cementing calcite has an almost pure endmember composition (Cal 97\%, Mgs 3\%, Sd $<1 \%$ ).

In the Basal Serpentinite, the HP $\operatorname{Atg}(1)^{\text {BS }}$ has high $\mathrm{Mg \#}$ ( $95-97 \%$ ) and $\mathrm{Al}$ (a.p.f.u.) 0.04-0.12 (Fig. 9a). The difference in $\mathrm{Mg \#}$ and $\mathrm{Al}$ (p.f.u.) in LSZ and Basal Serpentinite reflects the different bulk rock composition (section 6.1.1 and Fig. 5c). Olivine grown in equilibrium with $\operatorname{Atg}(1)^{B S}$ along the HP foliation has Mg\# 85-90\% (Fig. 9b). Metamorphic diopside and brucite have Mg\# 96-97 and 95, respectively.

The HP olivine and Ti-clinohumite veins in the Basal Serpentinite display two generations of olivine with different compositions (Fig. 4g). Olivine(1) has Mg\# 86-90\% and it is comparable with olivine in mylonitic foliation (Fig. 9b). Olivine(2) has a Mg\# ( 96\%) that is higher than that of olivine(1), and its $\mathrm{Mg \#}$ is like that of coexisting antigorite (Mg\# 96-97 and $\mathrm{Al} \sim 0.08-0.10$ a.p.f.u.; Fig. 9b) and diopside (Mg\# 97-98\%).

Ti-chondrodite and Ti-clinohumite (Fig. 9c-d) occur in both veins (\#16 and \#17) and along the HP foliation (\#23). Ti-chondrodite has $\mathrm{Ti}$ (a.p.f.u.) 0.20-0.35 and its composition is comparable to Ti-chondrodite found in the Zermatt-Saas Zone (Gilio et al., 2019). Ti-clinohumite has a Ti(a.p.f.u.) 0.25-0.5.

Table 4

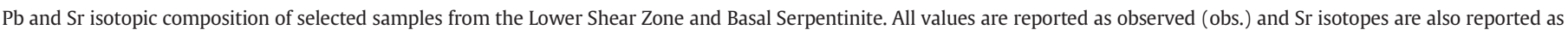
corrected for an age of $40 \mathrm{Ma}$. The age correction of $\mathrm{Pb}$ has been attempted but resulted $<$ than the given $2 \sigma$ error for each sample.

\begin{tabular}{|c|c|c|c|c|c|c|c|c|c|c|c|}
\hline & \multirow[t]{2}{*}{ Sample name } & \multirow[t]{2}{*}{ Rock type } & \multicolumn{2}{|c|}{${ }^{206} \mathrm{~Pb} /{ }^{204} \mathrm{~Pb}$} & \multicolumn{2}{|c|}{${ }^{207} \mathrm{~Pb} /{ }^{204} \mathrm{~Pb}$} & \multicolumn{2}{|c|}{${ }^{208} \mathrm{~Pb} /{ }^{204} \mathrm{~Pb}$} & \multicolumn{3}{|l|}{${ }^{87} \mathrm{Sr} /{ }^{86} \mathrm{Sr}$} \\
\hline & & & Meas. & $2 \sigma$ mean & Meas. & $2 \sigma$ mean & Meas. & $2 \sigma$ mean & Meas. & corr. & $2 \sigma$ mean \\
\hline \multirow[t]{5}{*}{ Basal serpentinite } & VI1304 & Static serpentinite & 17.916 & 0.029 & 15.471 & 0.021 & 37.677 & 0.056 & 0.706903 & 0.706832 & 0.000013 \\
\hline & GLSZ1417 & HP serpentinite & 18.689 & 0.001 & 15.636 & 0.001 & 38.651 & 0.001 & 0.707366 & 0.707362 & 0.000005 \\
\hline & GLSZ1410B & Olivine vein & 18.218 & 0.027 & 15.534 & 0.024 & 37.968 & 0.058 & 0.704895 & 0.704890 & 0.000008 \\
\hline & GLSZ1416 & Ti-clinohumite vein & 17.964 & 0.008 & 15.570 & 0.006 & 37.875 & 0.017 & 0.706397 & 0.706353 & 0.000008 \\
\hline & GLSZ1418 & Ti-clinohumite vein & 18.691 & 0.009 & 15.629 & 0.007 & 38.636 & 0.018 & n.d. & - & - \\
\hline \multirow[t]{3}{*}{ LSZ } & GLSZ1402 & Serpentinite & 18.162 & 0.011 & 15.614 & 0.010 & 38.138 & 0.024 & 0.707161 & 0.707128 & 0.000006 \\
\hline & GLSZ1411 & Serpentinite & 18.052 & 0.015 & 15.585 & 0.012 & 37.989 & 0.030 & 0.709562 & 0.709316 & 0.000498 \\
\hline & GLSZ1425 & Serpentinite & 18.117 & 0.005 & 15.618 & 0.004 & 38.109 & 0.010 & n.d. & - & - \\
\hline
\end{tabular}



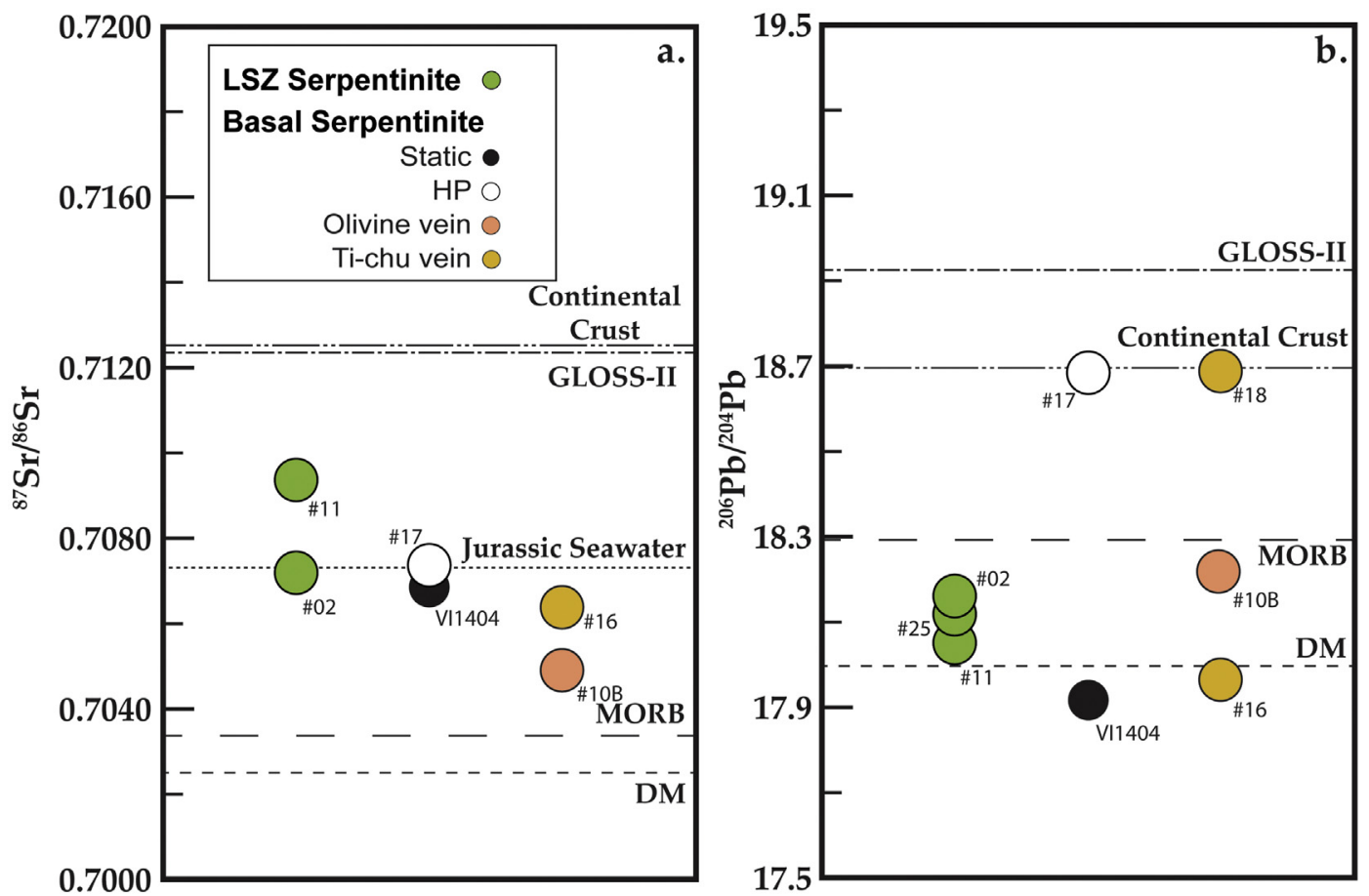

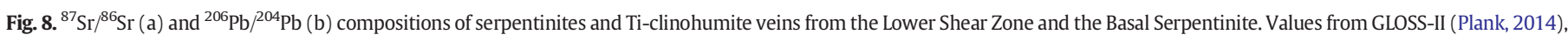

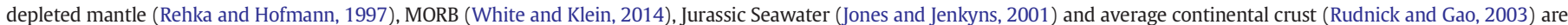
reported for comparison.

Table 5

Representative electron microprobe analyses of serpentinite from the Lower Shear Zone and the Basal serpentinite.

\begin{tabular}{|c|c|c|c|c|c|c|c|c|c|c|c|c|c|c|}
\hline \multirow{5}{*}{$\begin{array}{l}\frac{\text { Rock-type }}{\text { Sample }} \\
\text { SiO2 }\end{array}$} & \multicolumn{6}{|c|}{ Lower shear zone serpentinite } & \multicolumn{8}{|c|}{ Basal serpentinite } \\
\hline & \multicolumn{6}{|c|}{ Serpentinite } & \multicolumn{8}{|c|}{ Serpentinite } \\
\hline & \multicolumn{4}{|c|}{ GLSZ14-01 } & \multirow{2}{*}{\multicolumn{2}{|c|}{$\frac{\text { GLSZ14-23 }}{\text { Serpentine }}$}} & \multicolumn{8}{|c|}{ GLSZ1423 } \\
\hline & \multicolumn{2}{|c|}{ Serpentine (1) } & \multicolumn{2}{|c|}{ Serpentine (2) } & & & \multicolumn{2}{|l|}{ Olivine } & \multicolumn{2}{|c|}{ Serpentine } & \multicolumn{2}{|c|}{ Diopside } & \multicolumn{2}{|c|}{ Brucite } \\
\hline & 40.78 & 41.86 & 41.82 & 41.26 & 44.35 & 42.89 & 39.94 & 40.11 & 44.35 & 42.89 & 54.75 & 54.93 & 0.06 & 0.05 \\
\hline $\mathrm{TiO} 2$ & 0.01 & 0.02 & 0.00 & 0.00 & 0.00 & 0.00 & 0.01 & 0.05 & 0.00 & 0.00 & 0.00 & 0.00 & 0.00 & 0.01 \\
\hline $\mathrm{Al} 2 \mathrm{O} 3$ & 2.64 & 2.28 & 2.17 & 2.12 & 0.35 & 1.86 & 0.03 & 0.01 & 0.35 & 1.86 & 0.01 & 0.01 & 0.02 & 0.02 \\
\hline $\mathrm{Cr} 2 \mathrm{O} 3$ & 0.67 & 0.63 & 0.34 & 0.30 & 0.01 & 0.26 & 0.10 & 0.00 & 0.01 & 0.26 & 0.00 & 0.02 & 0.00 & 0.01 \\
\hline $\mathrm{FeO}$ & 7.40 & 7.29 & 7.36 & 7.19 & 3.19 & 3.49 & 14.12 & 13.97 & 3.19 & 3.49 & 1.23 & 1.29 & 7.55 & 7.20 \\
\hline $\mathrm{MnO}$ & 0.14 & 0.04 & 0.16 & 0.08 & 0.08 & 0.06 & 0.66 & 0.70 & 0.08 & 0.06 & 0.08 & 0.01 & 0.14 & 0.08 \\
\hline $\mathrm{MgO}$ & 34.62 & 34.55 & 34.86 & 34.73 & 38.72 & 38.13 & 46.16 & 46.10 & 38.72 & 38.13 & 18.56 & 17.78 & 65.47 & 65.83 \\
\hline $\mathrm{NiO}$ & 0.23 & 0.25 & 0.27 & 0.20 & 0.01 & 0.09 & 0.20 & 0.05 & 0.01 & 0.09 & 0.00 & 0.07 & 0.07 & 0.17 \\
\hline $\mathrm{CaO}$ & 0.07 & 0.00 & 0.00 & 0.01 & 0.00 & 0.00 & 0.02 & 0.04 & 0.00 & 0.00 & 25.20 & 25.47 & 0.01 & 0.00 \\
\hline $\mathrm{Na} 2 \mathrm{O}$ & 0.00 & 0.00 & 0.00 & 0.00 & 0.00 & 0.00 & 0.00 & 0.03 & 0.00 & 0.00 & 0.00 & 0.01 & 0.02 & 0.00 \\
\hline Total & 86.57 & 86.96 & 86.99 & 85.90 & 86.71 & 86.81 & 101.25 & 101.09 & 86.71 & 86.81 & 99.84 & 99.60 & 73.34 & 73.37 \\
\hline $\mathrm{Si}$ & 1.966 & 2.002 & 2.000 & 1.997 & 2.073 & 2.011 & 0.987 & 0.992 & 2.073 & 2.011 & 1.981 & 1.999 & 0.001 & 0.001 \\
\hline $\mathrm{Ti}$ & 0.000 & 0.001 & 0.000 & 0.000 & 0.000 & 0.000 & 0.000 & 0.001 & 0.000 & 0.000 & 0.000 & 0.000 & 0.000 & 0.000 \\
\hline $\mathrm{Al}$ & 0.150 & 0.129 & 0.122 & 0.121 & 0.019 & 0.103 & 0.001 & 0.000 & 0.019 & 0.103 & 0.000 & 0.001 & 0.000 & 0.000 \\
\hline $\mathrm{Cr}$ & 0.026 & 0.024 & 0.013 & 0.012 & 0.000 & 0.010 & 0.002 & 0.000 & 0.000 & 0.010 & 0.000 & 0.000 & 0.000 & 0.000 \\
\hline $\mathrm{Fe}(\mathrm{III})$ & 0.000 & 0.000 & 0.000 & 0.000 & 0.000 & 0.000 & 0.007 & 0.005 & 0.000 & 0.000 & 0.001 & 0.000 & 0.000 & 0.000 \\
\hline $\mathrm{Fe}(\mathrm{II})$ & 0.298 & 0.292 & 0.294 & 0.291 & 0.125 & 0.137 & 0.285 & 0.284 & 0.125 & 0.137 & 0.036 & 0.039 & 0.061 & 0.058 \\
\hline $\mathrm{Mn}$ & 0.006 & 0.002 & 0.006 & 0.003 & 0.003 & 0.002 & 0.014 & 0.015 & 0.003 & 0.002 & 0.002 & 0.000 & 0.001 & 0.001 \\
\hline $\mathrm{Mg}$ & 2.488 & 2.463 & 2.485 & 2.505 & 2.697 & 2.665 & 1.700 & 1.700 & 2.697 & 2.665 & 1.001 & 0.964 & 0.935 & 0.939 \\
\hline $\mathrm{Ni}$ & 0.009 & 0.009 & 0.011 & 0.008 & 0.000 & 0.003 & 0.004 & 0.001 & 0.000 & 0.003 & 0.000 & 0.002 & 0.001 & 0.001 \\
\hline $\mathrm{Ca}$ & 0.003 & 0.000 & 0.000 & 0.001 & 0.000 & 0.000 & 0.000 & 0.001 & 0.000 & 0.000 & 0.977 & 0.993 & 0.000 & 0.000 \\
\hline $\mathrm{Na}$ & 0.000 & 0.000 & 0.000 & 0.000 & 0.000 & 0.000 & 0.000 & 0.002 & 0.000 & 0.000 & 0.000 & 0.001 & 0.000 & 0.000 \\
\hline $\mathrm{H}$ & 4.000 & 4.000 & 4.000 & 4.000 & 4.000 & 4.000 & 0.000 & 0.000 & 4.000 & 4.000 & 0.000 & 0.000 & 2.001 & 2.000 \\
\hline Totals & 8.946 & 8.921 & 8.933 & 8.938 & 8.918 & 8.933 & 3.000 & 3.000 & 8.918 & 8.933 & 4.000 & 4.000 & 3.000 & 2.999 \\
\hline $\mathrm{Al} \#$ & 0.854 & 0.843 & 0.905 & 0.913 & 0.986 & 0.914 & 0.271 & 0.840 & 0.986 & 0.914 & 1.000 & 0.514 & 1.000 & 0.702 \\
\hline Mg\# & 0.893 & 0.894 & 0.894 & 0.896 & 0.956 & 0.951 & 0.856 & 0.857 & 0.956 & 0.951 & 0.966 & 0.961 & 0.939 & 0.942 \\
\hline
\end{tabular}

$\mathrm{Mg} \#=\mathrm{Mg} /\left(\mathrm{Fe}^{2+}+\mathrm{Mg}\right) ; \mathrm{Al} \#=\mathrm{Al} /\left(\mathrm{Al}+\mathrm{Cr}+\mathrm{Fe}^{3+}\right) ; \mathrm{H}$ and $\mathrm{Fe}^{3+}$ calculated assuming full site occupancy. 
Table 6

Representative electron microprobe analyses of olivine and of Ti-clinohumite veins.

\begin{tabular}{|c|c|c|c|c|c|c|c|c|c|c|c|c|c|c|c|c|c|c|c|c|c|c|c|c|c|}
\hline \multirow{5}{*}{$\begin{array}{l}\frac{\text { Rock-type }}{\text { Sample }} \\
\text { SiO2 }\end{array}$} & \multicolumn{25}{|c|}{ Basal serpentinite } \\
\hline & \multicolumn{14}{|c|}{ Sample GLSZ14 - olivine vein } & \multicolumn{11}{|c|}{ Sample GLSZ1416 - Ti-chondrodite vein } \\
\hline & \multicolumn{2}{|c|}{ Olivine (1) } & \multicolumn{2}{|c|}{ Olivine (2) } & \multicolumn{2}{|c|}{ Serpentine } & \multicolumn{2}{|c|}{ Diopside } & \multicolumn{2}{|c|}{ Ti-Chondrodite } & \multicolumn{2}{|c|}{ Ti-Clinohumite } & \multicolumn{2}{|c|}{ Magnetite } & \multicolumn{2}{|c|}{ Ti-chondrodite } & \multicolumn{2}{|c|}{ Ti-clinohumite } & \multicolumn{2}{|l|}{ Olivine } & \multicolumn{4}{|l|}{ Chlorite } & \multirow[t]{2}{*}{ Diopside } \\
\hline & & & & & & & & & & & & & & & & & & & & & Core & & Rim & & \\
\hline & 40.88 & 40.99 & 41.53 & 41.68 & 42.63 & 43.12 & 55.39 & 55.09 & 34.21 & 34.29 & 36.41 & 36.79 & 0.06 & 0.06 & 33.79 & 33.06 & 35.82 & 36.09 & 41.61 & 41.29 & 32.12 & 31.75 & 33.63 & 31.83 & 55.64 \\
\hline TiO2 & 0.06 & 0.00 & 0.18 & 0.06 & 0.05 & 0.01 & 0.00 & 0.00 & 7.06 & 6.30 & 3.66 & 3.43 & 0.10 & 0.00 & 7.29 & 7.11 & 3.59 & 3.56 & 0.05 & 0.00 & 0.00 & 0.00 & 0.00 & 0.00 & 0.07 \\
\hline Al203 & 0.01 & 0.00 & 0.02 & 0.02 & 1.89 & 1.45 & 0.02 & 0.00 & 0.04 & 0.04 & 0.01 & 0.00 & 0.00 & 0.00 & 0.00 & 0.00 & 0.00 & 0.01 & 0.02 & 0.03 & 16.81 & 16.94 & 13.71 & 13.76 & 0.04 \\
\hline Cr2O3 & 0.00 & 0.00 & 0.00 & 0.02 & 0.41 & 0.39 & 0.03 & 0.03 & 0.00 & 0.05 & 0.01 & 0.00 & 0.71 & 0.03 & 0.04 & 0.00 & 0.00 & 0.07 & 0.00 & 0.01 & 0.00 & 0.03 & 0.02 & 0.00 & 0.00 \\
\hline $\mathrm{FeO}$ & 8.76 & 8.96 & 4.10 & 4.07 & 2.62 & 2.20 & 0.92 & 0.71 & 6.56 & 7.62 & 8.55 & 8.26 & 91.63 & 92.45 & 9.24 & 9.25 & 11.94 & 11.91 & 9.10 & 9.32 & 6.53 & 6.54 & 3.55 & 3.64 & 1.22 \\
\hline MnO & 1.23 & 1.23 & 1.04 & 1.03 & 0.09 & 0.16 & 0.08 & 0.03 & 1.86 & 2.09 & 0.96 & 0.93 & 0.41 & 0.22 & 0.21 & 0.24 & 0.19 & 0.22 & 0.34 & 0.18 & 0.05 & 0.14 & 0.04 & 0.05 & 0.00 \\
\hline MgO & 49.33 & 49.16 & 53.20 & 53.22 & 38.29 & 39.10 & 17.96 & 18.04 & 47.51 & 46.80 & 48.17 & 48.68 & 0.78 & 1.04 & 46.17 & 46.15 & 46.00 & 45.82 & 49.02 & 49.04 & 30.75 & 30.76 & 34.23 & 33.66 & 17.67 \\
\hline $\mathrm{NiO}$ & 0.16 & 0.16 & 0.13 & 0.09 & 0.22 & 0.07 & 0.02 & 0.02 & 0.11 & 0.17 & 0.15 & 0.16 & 0.39 & 0.20 & 0.27 & 0.25 & 0.18 & 0.27 & 0.35 & 0.29 & 0.01 & 0.01 & 0.19 & 0.15 & 0.02 \\
\hline $\mathrm{CaO}$ & 0.02 & 0.02 & 0.01 & 0.04 & 0.00 & 0.00 & 25.28 & 25.58 & 0.03 & 0.02 & 0.03 & 0.00 & 0.03 & 0.00 & 0.02 & 0.02 & 0.00 & 0.02 & 0.03 & 0.02 & 0.00 & 0.00 & 0.01 & 0.02 & 25.37 \\
\hline $\mathrm{Na} 2 \mathrm{O}$ & 0.00 & 0.00 & 0.02 & 0.02 & 0.00 & 0.03 & 0.02 & 0.04 & 0.00 & 0.00 & 0.04 & 0.00 & 0.03 & 0.00 & 0.00 & 0.00 & 0.00 & 0.00 & 0.00 & 0.02 & 0.02 & 0.01 & 0.00 & 0.03 & 0.02 \\
\hline Total & 100.45 & 100.56 & 100.22 & 100.25 & 86.22 & 86.57 & 99.72 & 99.58 & 97.39 & 97.41 & 98.00 & 98.25 & 94.17 & 94.00 & 97.04 & 96.09 & 97.77 & 97.97 & 100.52 & 100.21 & 86.33 & 86.23 & 85.41 & 83.15 & 100.11 \\
\hline $\mathrm{Si}$ & 0.997 & 1.000 & 0.993 & 0.996 & 2.007 & 2.017 & 2.007 & 2.000 & 2.037 & 2.048 & 3.972 & 3.993 & 0.002 & 0.002 & 2.034 & 2.006 & 3.967 & 3.992 & 1.011 & 1.007 & 6.177 & 6.126 & 6.460 & 6.278 & 2.011 \\
\hline $\mathrm{Ti}$ & 0.001 & 0.000 & 0.003 & 0.001 & 0.002 & 0.000 & 0.000 & 0.000 & 0.316 & 0.283 & 0.300 & 0.280 & 0.003 & 0.000 & 0.330 & 0.324 & 0.299 & 0.296 & 0.001 & 0.000 & 0.000 & 0.000 & 0.000 & 0.000 & 0.002 \\
\hline $\mathrm{Al}$ & 0.000 & 0.000 & 0.001 & 0.001 & 0.105 & 0.080 & 0.001 & 0.000 & 0.003 & 0.003 & 0.001 & 0.000 & 0.000 & 0.000 & 0.000 & 0.000 & 0.000 & 0.002 & 0.000 & 0.001 & 3.811 & 3.852 & 3.104 & 3.199 & 0.002 \\
\hline $\mathrm{Cr}$ & 0.000 & 0.000 & 0.000 & 0.000 & 0.015 & 0.014 & 0.001 & 0.001 & 0.000 & 0.002 & 0.001 & 0.000 & 0.021 & 0.001 & 0.002 & 0.000 & 0.000 & 0.006 & 0.000 & 0.000 & 0.000 & 0.005 & 0.003 & 0.000 & 0.000 \\
\hline $\mathrm{Fe}(\mathrm{III})$ & 0.001 & 0.000 & 0.001 & 0.000 & 0.000 & 0.000 & 0.000 & 0.000 & 0.000 & 0.000 & 0.000 & 0.000 & 1.970 & 1.995 & 0.000 & 0.000 & 0.000 & 0.000 & 0.000 & 0.000 & 0.093 & 0.061 & 0.014 & 0.000 & 0.000 \\
\hline $\mathrm{Fe}(\mathrm{II})$ & 0.178 & 0.183 & 0.081 & 0.081 & 0.103 & 0.086 & 0.028 & 0.022 & 0.327 & 0.381 & 0.780 & 0.750 & 0.931 & 0.930 & 0.465 & 0.469 & 1.106 & 1.102 & 0.185 & 0.190 & 0.957 & 0.994 & 0.556 & 0.700 & 0.037 \\
\hline $\mathrm{Mn}$ & 0.025 & 0.025 & 0.021 & 0.021 & 0.004 & 0.006 & 0.003 & 0.001 & 0.094 & 0.106 & 0.089 & 0.086 & 0.013 & 0.007 & 0.011 & 0.013 & 0.018 & 0.021 & 0.007 & 0.004 & 0.009 & 0.022 & 0.006 & 0.008 & 0.000 \\
\hline Mg & 1.794 & 1.788 & 1.897 & 1.896 & 2.687 & 2.727 & 0.970 & 0.977 & 4.217 & 4.167 & 7.833 & 7.877 & 0.044 & 0.059 & 4.143 & 4.174 & 7.594 & 7.556 & 1.776 & 1.783 & 8.816 & 8.847 & 9.801 & 9.896 & 0.952 \\
\hline $\mathrm{Ni}$ & 0.003 & 0.003 & 0.003 & 0.002 & 0.008 & 0.003 & 0.001 & 0.000 & 0.005 & 0.008 & 0.013 & 0.014 & 0.012 & 0.006 & 0.013 & 0.012 & 0.016 & 0.024 & 0.007 & 0.006 & 0.001 & 0.002 & 0.030 & 0.023 & 0.001 \\
\hline $\mathrm{Ca}$ & 0.000 & 0.000 & 0.000 & 0.001 & 0.000 & 0.000 & 0.982 & 0.995 & 0.002 & 0.001 & 0.003 & 0.000 & 0.001 & 0.000 & 0.001 & 0.001 & 0.000 & 0.002 & 0.001 & 0.001 & 0.000 & 0.000 & 0.002 & 0.005 & 0.982 \\
\hline $\mathrm{Na}$ & 0.000 & 0.000 & 0.001 & 0.001 & 0.000 & 0.003 & 0.001 & 0.003 & 0.000 & 0.000 & 0.008 & 0.000 & 0.002 & 0.000 & 0.000 & 0.000 & 0.000 & 0.000 & 0.000 & 0.001 & 0.012 & 0.005 & 0.000 & 0.020 & 0.001 \\
\hline $\mathrm{H}$ & 0.000 & 0.000 & 0.000 & 0.000 & 4.000 & 4.000 & 0.000 & 0.000 & & & & & 0.000 & 0.000 & & & & & & & 16.000 & 16.000 & 16.000 & 16.000 & \\
\hline Totals & 3.000 & 3.000 & 3.000 & 3.000 & 8.931 & 8.937 & 3.993 & 4.000 & 7.000 & 7.000 & 13.000 & 13.000 & 3.000 & 3.000 & 7.000 & 7.000 & 13.000 & 13.000 & 2.988 & 2.993 & 35.878 & 35.921 & 35.984 & 36.136 & 3.988 \\
\hline Al\# & 1.000 & - & 1.000 & 0.547 & 0.872 & 0.847 & 0.443 & 0.000 & 1.000 & 0.574 & 0.545 & 0.000 & 0.000 & 0.000 & 0.159 & - & - & 0.228 & 1.000 & 0.823 & 1.000 & 0.999 & 0.999 & 1.000 & 1.000 \\
\hline Mg\# & 0.910 & 0.907 & 0.959 & 0.959 & 0.963 & 0.969 & 0.972 & 0.978 & 0.928 & 0.916 & 0.909 & 0.913 & 0.045 & 0.059 & 0.899 & 0.899 & 0.873 & 0.873 & 0.906 & 0.904 & 0.902 & 0.899 & 0.946 & 0.934 & 0.963 \\
\hline
\end{tabular}




\section{Discussion}

\subsection{The evolutionary record of the LSZ and basal serpentinite}

\subsubsection{The LSZ}

Eclogites and metasediments from the LSZ still preserve some prograde-to-peak subduction-zone metamorphic assemblages, as shown by recent petrological studies (Angiboust et al., 2012a, 2012b; Groppo and Castelli, 2010; Locatelli et al., 2018). In contrast, the associated antigorite serpentinite mylonites are much less known. In the LSZ, serpentinites lack olivine and Ti-chondrodite/Ti-clinohumite, the typical eclogite-facies metamorphic minerals for serpentinite systems, which are not reported in the previous descriptions of the Monviso metaophiolite (except one mention in Compagnoni et al., 1980). Here we show that an $\operatorname{Atg}(2)^{\mathrm{LSZ}}$ mylonitic foliation envelops previous Atg $(1)^{\mathrm{LSZ}}$, reflecting successive crystallization stages of antigorite during a multi-stage tectonic history, as for nearby mafic eclogites (Angiboust et al., 2012b; Groppo and Castelli, 2010; Locatelli et al., 2018). The relative chronology of mineral crystallization in the LSZ serpentinites is summarized in Table 7.
An important observation is the occurrence of carbonate veins in the $\mathrm{LSZ}$ serpentinite. The mylonitic $\operatorname{Atg}(2)^{\mathrm{LSZ}}$ foliation transposes magnesite + talc-bearing veins. In such veins, magnesite is overgrown by dolomite and then by calcite-bearing assemblages, again documenting multiple replacement episodes. Magnesite replacement by dolomite and calcite has been described for several HP and UHP rocks and has been related to a decrease in P-T conditions during exhumation (Liu et al., 2002; Malaspina et al., 2009; Scambelluri et al., 2014; Scambelluri et al., 2016). HP magnesite veins cross-cutting an olivine + antigorite foliation in a HP serpentinite have been observed in the Voltri massif, and peak magnesite replaced by dolomite and calcite has been reported for the Cima di Gagnone Alpine metaperidotite (Scambelluri et al., 2014, 2016). While Montes-Hernandez et al. (2014) have shown that the magnesite-to-dolomite reaction can also occur at lower conditions $\left(T<200^{\circ} \mathrm{C}\right)$, the structural relationships, mineral assemblage and presence of Na-rich pyroxene around these veins (Angiboust et al., 2014) make this hypothesis unlikely. The mylonitic foliation in the LSZ serpentinites thus reworks previous eclogite-facies structures and assemblages formed in the serpentinite during its prograde and/or peak history and must be interpreted as retrograde (see Fig. 10a). At present, there is no quantitative PT estimate available for a pure magnesite-talc
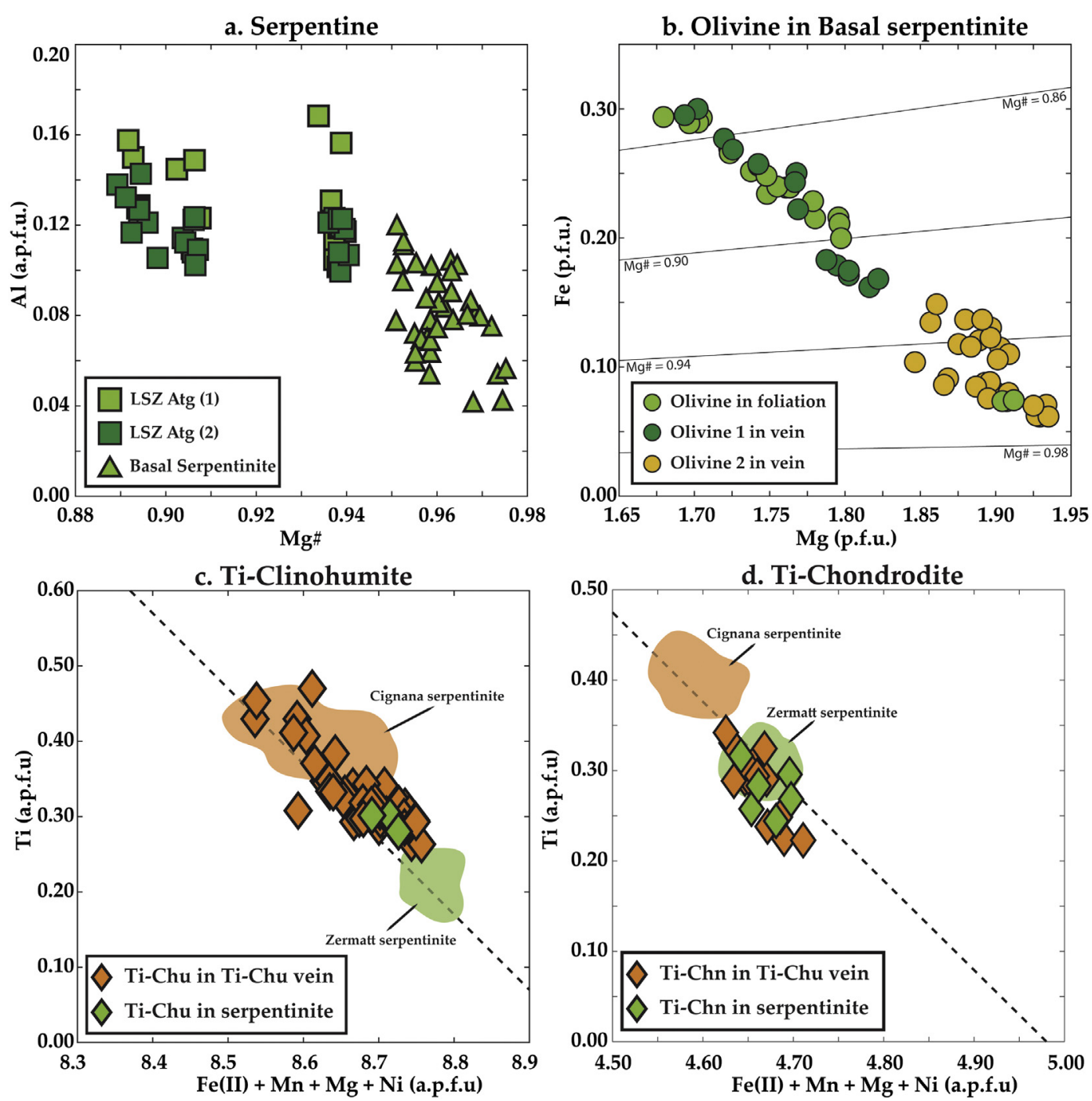

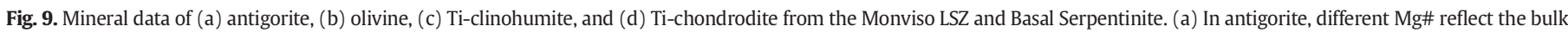

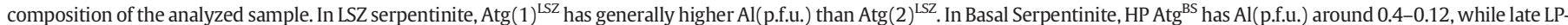

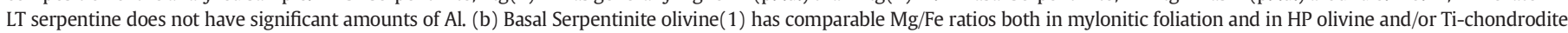

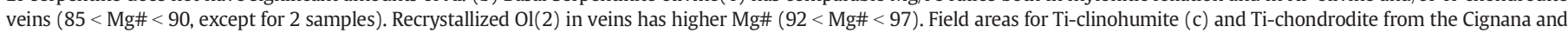
Zermatt serpentinites (Gilio et al., in review) are reported for comparison. 
hypothesis (2): the main $\operatorname{Atg}(2)^{\mathrm{LSZ}}$ foliation of the LSZ probably reworked and re-equilibrated a former olivine-bearing antigorite foliation. The PT conditions of $\operatorname{Atg}(2)^{\mathrm{LSZ}}$ formation in the LSZ serpentinite are shown by the green path in Fig. 10a, below the antigorite + brucite $=$ olivine $+\mathrm{H}_{2} \mathrm{O}$ reaction curve $\left(\mathrm{T}<\sim 450{ }^{\circ} \mathrm{C}\right)$. In this interpretation, formation of the pervasive mylonitic foliation across the LSZ can be ascribed to a continuous exhumation history, from peak eclogitefacies conditions to progressively shallower levels marked by the final greenschist-facies re-equilibration (green line in Fig. 10a; Schwartz et al., 2000; Angiboust et al., 2012b).

\subsubsection{The basal serpentinite}

Our textural and petrologic study indicates that the Basal Serpentinite of the LSU still preserves evidence of the prograde-topeak metamorphic evolution, as commonly observed for orogenic serpentinites. Undeformed serpentinite still preserves textures which can be attributed to a stage of oceanic serpentinization of a former oceanic peridotite (mesh structure, bastite; Fig. 4). This LP-LT hydration of the mantle protolith produces LT serpentine polymorphs lizardite and chrysotile, which usually become replaced by antigorite in subducted Alpine serpentinites (Cannaò et al., 2016; Debret et al., 2013; Scambelluri et al., 1995, 1997).

In the Basal Serpentinite, mesh textures and bastites are found as relictual microlithons embedded within the main antigorite \pm brucite foliation formed at the expense of chrysotile/lizardite during prograde metamorphism at $T<300^{\circ} \mathrm{C}$ (Schwartz et al., 2013). This stage therefore records early subduction of the ocean floor serpentinite (e.g. in a forearc setting; Kodolányi and Pettke, 2011) prior to peak eclogite-facies conditions (blue line in Fig. 10b). This prograde evolution is comparable to that described by Groppo and Castelli (2010) for lawsonite-eclogites of the LSU.

The peak metamorphic event is rather well preserved in the Basal Serpentinite and is represented by the formation of an antigorite + olivine mylonite fabric wrapping around the previous bastite structures (Fig. 4c-d). These mineral assemblages provide good constraints for temperature but not pressure, so that the exact PT path and peak burial conditions must be inferred from the associated eclogites (Fig. 10a-b; Angiboust et al., 2012b). The PT path for the LSZ eclogite crosses the antigorite + brucite $=$ olivine $+\mathrm{H}_{2} \mathrm{O}$ reaction curve at $\sim 500{ }^{\circ} \mathrm{C}$ and 2.3-2.5 GPa, providing a minimum $\mathrm{T}$ estimate for this metamorphic event. Brucite, however, remains stable for the entire metamorphic history. Bretscher et al. (2018) compiled brucite-out reaction curves for different reactive bulk compositions and found that highly-Mg brucite (Mg\# 95) is stable until about $550{ }^{\circ} \mathrm{C}$. This is consistent with peak T metamorphic conditions found in eclogite $\left(550-570{ }^{\circ} \mathrm{C}\right.$; Fig. 10a-b; Groppo and Castelli, 2010).

As discussed in Shen et al. (2015), Ti-chondrodite is a potential pressure indicator of transition to UHP conditions in hydrated ultramafic systems. In the Zermatt Saas serpentinite, Ti-chondrodite-bearing veins have recently been attributed to a stage of ultra-high pressure metamorphism reached by this serpentinite during Alpine subduction (Luoni et al., 2018). The peak metamorphic conditions of $500-550{ }^{\circ} \mathrm{C}$ and 2.5-2.7 GPa suggested for the LSZ eclogite Angiboust et al. (2012b) fall just above the Ti-clinohumite to Ti-chondrodite transition defined by Shen et al. (2015) the thick curve features the $\pm 0.2 \mathrm{GPa}$ uncertainty on this transition. The red arrow in Fig. 10b therefore constrains peak PT conditions for the Basal Serpentinite, from early olivine formation to the Ti-chondrodite stability field. While peak metamorphic conditions of the LSZ (and Basal Serpentinite) should fall slightly above the quartz-to-coesite transition line (Angiboust et al., 2012b; Locatelli et al., 2018), coesite was not (yet) found at Monviso, suggesting that overstep of this reaction may have not occurred.

The Ti content of Ti-chondrodite and Ti-clinohumite can vary with pressure (Lopez Sanchez-Vizcaino et al., 2005; Shen et al., 2015). Ti-chondrodite within the HP Basal Serpentinite has a Ti content comparable to that in the HP Zermatt-Saas Zone (Gilio et al., 2019; Luoni et al.,

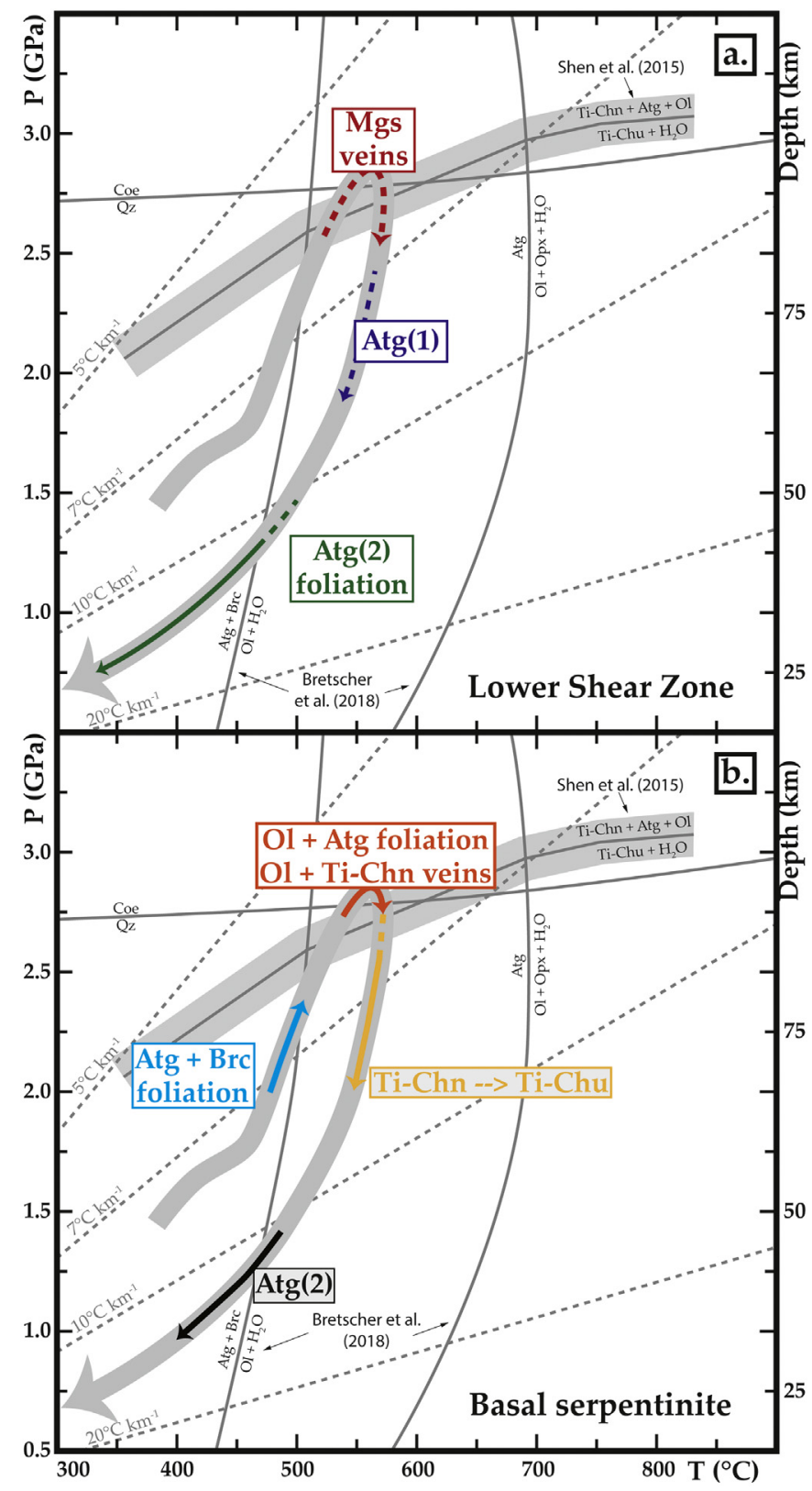

Fig. 10. (a) PT path in the Lower Shear Zone recorded in antigorite mylonites. Vein magnesite crystallization (dashed red line) and its overprint by dolomite and calcite (dashed blue line) are highlighted. Retrograde crystallization of $\operatorname{Atg}(1)$ and $A \operatorname{tg}(2)$ are illustrated. (b) PT path recorded in the Basal Serpentinite. The original PT path was proposed for eclogites by Angiboust et al. (2012b).

2018), consistent with the similar peak metamorphic conditions in both localities $\left(550{ }^{\circ} \mathrm{C}\right.$ and $2.3-2.7 \mathrm{GPa}$ and $500-550{ }^{\circ} \mathrm{C}$ and $2.5-2.7 \mathrm{GPa}$, for Zermatt and Monviso respectively). In some deformed veins, the original HP phases Ti-chondrodite and chlorite(1) recrystallize into Ti-clinohumite and chlorite(2), as documented for the Cignana serpentinites (Gilio et al., 2019). The crystallization of Ti-clinohumite at the expense of Ti-chondrodite can be ascribed to decreasing pressure conditions, hence to an initial stage of (isothermal) decompression experienced by the Basal Serpentinite (orange line in Fig. 10b).

The Basal Serpentinite (Table 7) therefore preserves evidence of its initial mantle assemblage, made of olivine, clinopyroxene, orthopyroxene (now bastite) and Al-rich phases such as spinel (now chlorite + magnetite), as well as hydration on the seafloor, as indicated by the presence of metarodingites, and then subduction to eclogite 
facies conditions, where metamorphic olivine formed by partial breakdown of brucite. Multiple sets of Ti-bearing veins also formed during and after development of the HP foliation (Fig. 10b). This multi-stage record preserved in the Basal Serpentinite, consistent with the one observed in nearby eclogites, strikingly contrasts with the record of the LSZ serpentinites, in which strain localization and retrogression completely obliterated the prograde-to-peak history.

\subsection{The geochemical evolution of LSZ and basal serpentinite}

In this section, we scrutinize major and trace element compositions to assess (1) whether the LSZ and Basal Serpentinite derive from a common mantle protolith, (2) the extent of fluid-rock interactions and equilibration with subduction zone fluids (and their origin) and, importantly, (3) the relative timing of juxtaposition of these serpentinites with other rock reservoirs.

\subsubsection{The mantle protolith}

The major and trace element composition of a pristine oceanic mantle peridotite is the result of a series of multiple events of depletion during partial melting and melt-rock interactions (Godard et al., 2000; Müntener et al., 2004; Rampone et al., 2004). The major element (except for CaO; Niu, 2004) and the REE budget of pristine peridotite frequently remains unaltered during serpentinization and fluid-rock interaction, making serpentinites useful tools to assess the geochemical and petrologic evolution of the oceanic mantle (see Deschamps et al., 2013, and references therein).

The very low $\mathrm{CaO}$ content of the Monviso serpentinite is incompatible with the $\mathrm{Al}_{2} \mathrm{O}_{3}-\mathrm{CaO}$ melt depletion evolution of peridotite (Fig. $5 \mathrm{a}$ ). The decrease in $\mathrm{CaO}$ contents of serpentinite with respect to the mantle depletion trend recorded by most Monviso serpentinites (Fig. 5a) is likely the effect of intense serpentinization leading to clinopyroxene replacement by oceanic serpentine along with a sharp drop in $\mathrm{CaO}$ contents. This suggests that (i) the original protolith of such serpentinites was a variably depleted harzburgite and (ii) the oceanic mantle composition at Monviso was rather homogeneous.

\subsubsection{The extent of fluid rock interactions in the LSU serpentinite}

The variability in trace element composition of Basal Serpentinite strongly correlates with textures. Sample VI13-04 (Basal Serpentinite "static" in Fig. 7 and Fig. 4a) is enriched in B and W with respect to depleted mantle values, typical for oceanic serpentinization (e.g., Bonatti et al., 1984; Kodolányi et al., 2012; Peters et al., 2017). It is only just slightly enriched in crust-derived fluid mobile elements such as As, $\mathrm{Sb}$, U, Ba and Cs (Cannaò et al., 2016; Debret et al., 2013; Lafay et al., 2013; Scambelluri et al., 1997). Moreover, its Sr isotopic signature is comparable to Jurassic seawater values and the $\mathrm{Pb}$ isotopic signature to DM values. Sample VI13-04 thus preserves an oceanic serpentinization signature, whose composition remained unchanged during subduction and related fluid-rock interactions.

The Basal Serpentinite samples showing both prograde and HP foliations (e.g. sample \#17) are characterized by a progressive increase in fluid mobile $\mathrm{As}, \mathrm{Sb}, \mathrm{Ba}$, and $\mathrm{Cs}$, from values analogous to the static serpentinite (sample VI13-04) towards the GLOSS-II and the LSZ oceanic metasediment compositions. Conversely, the B content is uniform (Basal Serpentinite "prograde" and "HP" in Fig. 7). This enrichment in fluid mobile trace elements (FME; As, Sb, Ba, and Cs) during subduction appears to be coupled with an increase in radiogenic $\mathrm{Pb}$ isotopic values observed in the olivine-bearing serpentinite towards GLOSS-II values (sample \#17; Fig. 8).

The olivine (sample \#10B) and Ti-clinohumite (samples \#16 and \#18) veins show contrasting levels of enrichment in FMEs (Table 2). The olivine vein is strongly enriched in $\mathrm{As}$ and $\mathrm{Sb}$ and depleted in $\mathrm{U}$ and $\mathrm{Th}$, and the Ti-clinohumite veins are depleted in $\mathrm{As}$ and $\mathrm{Sb}$ and enriched in $U$ and $T h$. This difference in trace element compositions between HP veins might be related to (1) heterogeneities in the composition of fluids circulating within the Basal Serpentinite at HP (i.e. evolving fluid composition and/or different fluid source) or (2) differences in compatibility of certain FMEs (such as As, Sb, U and Th) within phases contained in olivine and Ti-clinohumite veins. These HP veins also show contrasting $\mathrm{Pb}$ and $\mathrm{Sr}$ isotopic compositions, from values closer to DM towards values comparable with continental crust and/or oceanic metasediments (Fig. 8). Angiboust et al. (2014) described a similar variability in fluid composition during the prograde history of the Monviso metaophiolite: the 'fluid pulses' recorded in LSZ eclogite garnets point to different sources, from crustal to metasedimentary, possibly due to successive mineral dehydration reactions during burial.

The geochemical and isotopic enrichment trends observed in the prograde foliated serpentinite of the Basal Serpentinite are comparable to enrichments in FMEs described in other Alpine HP serpentinite and metaperidotite (e.g. Voltri and Cima di Gagnone), which have been related to interaction between serpentinite and metasediments during subduction (Cannaò et al., 2016; Scambelluri et al., 2014; Scambelluri et al., 2019). Prograde to peak burial fluid-rock interactions were not pervasive across the whole Basal Serpentinite, however, but rather concentrated along more deformed sections since some still retain their oceanic serpentinization signature.

In comparison, the LSZ shows more homogeneous geochemical and isotopic characteristics, with enrichments in FMEs such as As, Sb, U, Ba, and Cs similar to the most enriched Basal Serpentinite samples. The Srisotopic signature is also more enriched than Jurassic Seawater, similarly to what Cannaò et al., 2016 documented for the Voltri serpentinites. While the main mylonitic foliation of the LSZ serpentinite formed during exhumation, the presence of eclogite-facies magnesite veins, stretched and reworked by the mylonitic foliation, suggests that fluid rock interactions also occurred in the LSZ during peak metamorphic conditions. This is consistent with the report of HP metasomatic haloes around eclogite blocks enveloped in the LSZ (Angiboust et al., 2014). The final geochemical and isotopic homogenization observed in the LSZ might reflect intense syn-deformational fluid percolation and fluid-rock interaction during the blueschist to greenschist facies exhumation (Locatelli et al., 2018). This episode likely relates to the last pervasive deformation increments along the Monviso shear zones, which were recently constrained by multi-mineral $\mathrm{Rb}-\mathrm{Sr}$ dating near $\sim 36 \mathrm{Ma}$ and at $\sim 400{ }^{\circ} \mathrm{C}$ and $1 \mathrm{GPa}$, in the epidote-blueschist facies (S. Angiboust, Personal Communication).

\subsection{Tectonic and geophysical implications}

The textural-geochemical relationships documented in the Basal Serpentinite of the Lago Superiore Unit suggest that it first acquired an oceanic serpentinization signature, during Jurassic exposure on the seafloor, and later interacted with metasediment-derived fluids during the prograde subduction history, up to peak eclogite-facies metamorphism. The extent to which the lithospheric mantle is serpentinized at depth during subduction or rather during exposure on the seafloor is probably variable. Examples from Lanzo and Erro-Tobbio peridotite bodies suggest that large slices of oceanic lithosphere may diffusely preserve the structural and petrologic record of the mantle evolution through the Alpine subduction and exhumation history (Pelletier and Müntener, 2006; Scambelluri et al., 1995). Trace element and isotopic signatures acquired during seafloor hydration can be preserved in undeformed domains (e.g. sample VI13-04; Cannaò et al., 2016), as may the redox state up to antigorite dehydration (Bretscher et al., 2018), or modified through interaction with subduction-related fluids (Cannaò et al., 2015; Cannaò et al., 2016).

Our new geochemical data suggest that the serpentinized mantle of both the Basal Serpentinite and LSZ was infiltrated by slab fluids and enriched by sediment-derived FMEs during the prograde subduction stage down to eclogite facies conditions, as exemplified by the enriched 
olivine and Ti-clinohumite veins still preserved in the Basal Serpentinite (Fig. 8).

Fig. 11 illustrates a possible evolution of the LSU during peak metamorphic conditions. Angiboust et al. (2012b) suggested that the LSU detached from the down-going slab during the peak HP metamorphic event, near $80 \mathrm{~km}$ depth, and returned along the plate interface as a relatively coherent portion of oceanic lithosphere. This was accompanied by Intense deformation and brecciation during detachment and/or early exhumation, and massive fluid infiltration and metasomatism (Fig. 4a-b in Angiboust et al., 2012a). The base of the Basal Serpentinite itself represents the former décollement horizon along which the the LSU was detached from the slab (Locatelli et al., 2018), and was reworked during its juxtaposition with the underlying UHP DoraMaira continental massif. This might have allowed metasedimentderived fluids (blue arrows in Fig. 11), released by the metasedimentary cover of the Dora-Maira massif to move along the plate interface into the Basal Serpentinite. Whether this enrichment was caused by fluids derived from continental crust (i.e. the Dora Maira) or from the metasedimentary cover of the oceanic crust is unclear. Juxtaposition of the LSU with other slab fragments (e.g. the Monviso unit) may have indeed allowed sediment-derived fluids to move upward along tectonic contacts between the slices (such as the Basal Serpentinite) and within HP shear zones (such as the LSZ). The fact that most of the Basal Serpentinite preserved its prograde and peak assemblages and geochemical imprint, and thus escaped later deformation, indicates that strain was restricted to the bottom of the Basal Serpentinite near the contact with the Dora Maira Massif (blue dashed line in Fig. 11). In contrast, retrograde deformation affected the LSZ extensively and reset its geochemical signature, preserving eclogite facies textures and mineralogy only in magnesite veins and eclogite blocks.

In Alpine-type subduction zones where slow-spreading oceanic lithosphere subducts, subducted oceanic crust and sediments are relatively subordinate compared to partially hydrated mantle. The weak rheology of serpentinites and serpentinized peridotites (Hilairet et al., 2007) may thus dominate the physical and mechanical properties of the downgoing plate, controlling decoupling from the subducting slab and producing a cold and stagnant mantle wedge (Agard et al., 2018; Guillot et al., 2015; Wada and Wang, 2009). The LSU described here represents a "snap-shot" of Alpine metamorphic and shearing events, from prograde subduction to exhumation. Its km-scale thickness, and the oriented antigorite fabric in the Lower Shear Zone and Basal Serpentinite, which enhances seismic reflectivity (Watanabe et al., 2011), make it a potentially good seismic reflector. As such, the Lago Superiore Unit is probably an exhumed fragment of a paleo- "low velocity zone", that might have been like the ones observed in active subduction zones via seismic tomography. This example could be used as a proxy of a deep (70-80 km) Alpine-type subduction zone to better interpret seismic images of present-day convergent margins.

\section{Conclusions}

The Lago Superiore Unit at Monviso (W. Alps) is a slice of oceanic lithosphere which experienced eclogite facies metamorphism during the Alpine subduction event. The basal section of the LSU consists of oceanic serpentinites which were locally enriched by fluids of metasedimentary origin.

During exhumation, most deformation and mineral re-equilibration was localized in the LSZ, near the base of the $\mathrm{Mg}-\mathrm{Al}$ gabbro blocks. Fluid percolation during progressive exhumation chemically rehomogenized the serpentinites in the LSZ serpentinite, which retain its HP geochemical composition and mineralogy only within deformed HP magnesite veins. While most of the deformation during the eclogite-facies peak and retrograde history localized along the LSZ, the underlying Basal Serpentinite largely escaped such retrograde overprint, and thus still records sections of the prograde history of the LSU serpentinite, from ocean floor hydration to HP metamorphic conditions.

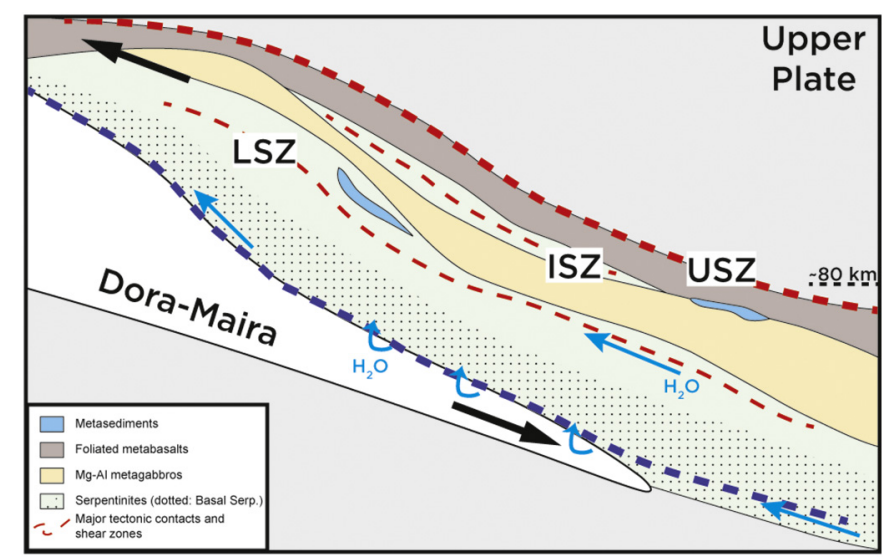

Fig. 11. Tectonic sketch showing the evolution of the Lago Superiore Unit at peak metamorphic conditions ( $\sim 80 \mathrm{~km}$ depth). The Lago Superiore Unit was an active plate interface environment during subduction, pinned out between the Upper Plate or another tectonic slice (USZ) and the subducting oceanic lithosphere. During exhumation, the LSU accreted on top of the subducting continental Dora-Maira unit (black arrows). This might have allowed metasediment-derived fluids (blue arrows) released by the metasedimentary cover of the Dora-Maira massif to move along the plate, into the Basal Serpentinite. Localized exhumation-related deformation was accommodated at the top of the serpentinite sliver, at the contact with the overlying metamafic crust forming the Lower Shear Zone. This deformation created a preferential pathway for metamorphic fluids, which pervasively reset the chemical composition of the LSZ serpentinite.

\section{Declaration of Competing Interest}

The authors declare that they have no known competing financial interests or personal relationships that could have appeared to influence the work reported in this paper.

\section{Acknowledgments}

We greatly benefitted from discussions with E. Cannaò, H. Marschall, J. Hermann, D. Rubatto, and all scientists and fellows from the ZIP project (http://www.zip-itn.eu/): discussions within ZIP stimulated this work. We thank the two anonymous reviewers and the Editor César Ranero for their comments and suggestions which helped improving the first version of this manuscript. We also thank A. Risplendente and L. Negretti for technical assistance during the SEM and wavelengthdispersive spectrometry microprobe work. MG has received funding from the European Research Council (ERC) under the European Union's Horizon 2020 research and innovation programme (grant agreement No 714936 for the project TRUE DEPTHS to M. Alvaro). M.G., M.S., T.P., M.L. and P.A. acknowledge funding by the People Programme (Marie Curie Actions, European Union's Seventh Framework Programme FP7/ 2007-2013) to the Initial Training Network ZIP (Zooming In-between Plates, REA grant agreement no. 604713). M.S. also acknowledges support from the Italian MIUR and the University of Genova.

\section{Appendix A. Supplementary data}

Supplementary data to this article can be found online at https://doi. org/10.1016/j.lithos.2019.105308.

\section{References}

Agard, P., Yamato, P., Jolivet, L., Burov, E., 2009. Exhumation of oceanic blueschists and eclogites in subduction zones: timing and mechanisms. Earth-Sci. Rev. 92, 53-79.

Agard, P., Plunder, A., Angiboust, S., Bonnet, G., Ruh, J., 2018. The subduction plate interface: rock record and mechanical coupling (from long to short time scales). Lithos. 320, 537-566.

Angiboust, S., Agard, P., Raimbourg, H., Yamato, P., Huet, B., 2011. Subduction interface processes recorded by eclogite-facies shear zones (Monviso, W. Alps). Lithos 127, 222-238. 
Angiboust, S., Agard, P., Yamato, P., Raimbourg, H., 2012a. Eclogite breccias in a subducted ophiolite: a record of intermediate-depth earthquakes? Geology 40, 707-710.

Angiboust, S., Langdon, R., Agard, P., Waters, D., Chopin, C., 2012b. Eclogitization of the Monviso ophiolite (W. Alps) and implications on subduction dynamics. J. Metamorph. Geol. 30, 37-61.

Angiboust, S., Wolf, S., Burov, E., Agard, P., Yamato, P., 2012c. Effect of fluid circulation on subduction interface tectonic processes: Insights from thermo-mechanical numerical modelling. Earth Planet. Sci. Lett. 357, 238-248.

Angiboust, S., Pettke, T., De Hoog, J.C., Caron, B., Oncken, O., 2014. Channelized fluid flow and eclogite-facies metasomatism along the subduction shear zone. J. Petrol. 55 883-916.

Auzende, A.-L., Guillot, S., Devouard, B., Baronnet, A., 2006. Serpentinites in an Alpine convergent setting: effects of metamorphic grade and deformation on microstructures. Eur. J. Mineral. 18, 21-33.

Balestro, G., Lombardo, B., Vaggelli, G., Borghi, A., Festa, A., Gattiglio, M., 2014 Tectonostratigraphy of the northern Monviso meta-ophiolite complex (western Alps). Ital. J. Geosci. 133, 409-426.

Balestro, G., Festa, A., Dilek, Y., Tartarotti, P., 2015. Pre-Alpine extensional tectonics of peridotite-localized oceanic core complex in the late Jurassic, high-pressure Monviso ophiolite (Western Alps). Episodes 38, 266-282

Balestro, G., Festa, A., Borghi, A., Castelli, D., Gattiglio, M., Tartarotti, P., 2018. Role of late Jurassic intra-oceanic structural inheritance in the Alpine tectonic evolution of the Monviso meta-ophiolite complex (Western Alps). Geol. Mag. 155, 233-249.

Bebout, G.E., 2007. Metamorphic chemical geodynamics of subduction zones. Earth Planet. Sci. Lett. 260, 373-393.

Blake Jr., M., Moore, D., Jayko, A., 1995. The role of serpentinite melanges in the unroofing of UHPM rocks: an example from the Western Alps of Italy. Ultrahigh Pressure Metamorphism, pp. 182-205.

Bonatti, E., Lawrence, J.R., Morandi, N., 1984. Serpentinization of oceanic peridotites: temperature dependence of mineralogy and boron content. Earth Planet. Sci. Lett. 70, 88-94.

Bostock, M., Hyndman, R., Rondenay, S., Peacock, S., 2002. An inverted continental Moho and serpentinization of the forearc mantle. Nature 417, 536

Breeding, C.M., Ague, J.J., Bröcker, M., 2004. Fluid-metasedimentary rock interactions in subduction-zone mélange: implications for the chemical composition of arc magmas. Geology 32, 1041-1044.

Bretscher, A., Hermann, J., Pettke, T., 2018. The influence of oceanic oxidation on serpentinite dehydration during subduction. Earth Planet. Sci. Lett. 499, 173-184.

Brovarone, A.V., Beltrando, M., Malavieille, J., Giuntoli, F., Tondella, E., Groppo, C., Beyssac, O., Compagnoni, R., 2011. Inherited ocean-continent transition zones in deeply subducted terranes: insights from Alpine Corsica. Lithos 124, 273-290.

Cannaò, E., Agostini, S., Scambelluri, M., Tonarini, S., Godard, M., 2015. B, Sr and Pb isotope geochemistry of high-pressure Alpine metaperidotites monitors fluid-mediated element recycling during serpentinite dehydration in subduction mélange (Cima di Gagnone, Swiss Central Alps). Geochim. Cosmochim. Acta 163, 80-100.

Cannaò, E., Scambelluri, M., Agostini, S., Tonarini, S., Godard, M., 2016. Linking serpentinite geochemistry with tectonic evolution at the subduction plateinterface: the Voltri Massif case study (Ligurian Western Alps, Italy). Geochim. Cosmochim. Acta 190, 115-133.

Castelli, D., Lombardo, B., 2007. The plagiogranite-FeTi-oxide gabbro association of Verne (Monviso metamorphic ophiolite, western Alps). Ofioliti 32, 1-14.

Castelli, D., Rostagno, C., Lombardo, B., 2002. Jd-Qtz-bearing metaplagiogranite from the Monviso meta-ophiolite (Western Alps). Ofioliti 27, 81-90.

Chalot-Prat, F., Ganne, J., Lombard, A., 2003. No significant element transfer from the oceanic plate to the mantle wedge during subduction and exhumation of the Tethys lithosphere (Western Alps). Lithos 69, 69-103.

Cliff, R., Barnicoat, A., Inger, S., 1998. Early Tertiary eclogite facies metamorphism in the Monviso Ophiolite. J. Metamorph. Geol. 16, 447-455.

Compagnoni, R., Fiora, L., Lombardo, B., Messiga, B., Nervo, R., Piccardo, G.B., 1980. Excursion I: The Monviso Ophiolite Complex - Cottian Alps. Ophiolites : Proceedings, International Ophiolite Symposium, Cyprus, 1979.

Compagnoni, R., Rolfo, F., Manavella, F., Salusso, F., 2007. Jadeitite in the Monviso metaophiolite, Piemonte zone Italian. Western Alps. Per. Mineral. 76, 79-89.

Debret, B., Nicollet, C., Andreani, M., Schwartz, S., Godard, M., 2013. Three steps of serpentinization in an eclogitized oceanic serpentinization front (Lanzo MassifWestern Alps). J. Metamorph. Geol. 31, 165-186.

Deschamps, F., Godard, M., Guillot, S., Hattori, K., 2013. Geochemistry of subduction zone serpentinites: a review. Lithos $178,96-127$.

Duchêne, S., Blichert-Toft, J., Luais, B., Télouk, P., Lardeaux, J.-M., Albarède, F., 1997. The Lu-Hf dating of garnets and the ages of the Alpine high-pressure metamorphism. Nature 387, 586.

Festa, A., Balestro, G., Dilek, Y., Tartarotti, P., 2015. A Jurassic oceanic core complex in the high-pressure Monviso ophiolite (western Alps, NW Italy). Lithosphere 7, 646-652.

Gerya, T.V., Stöckhert, B., Perchuk, A.L., 2002. Exhumation of high-pressure metamorphic rocks in a subduction channel: a numerical simulation. Tectonics 21, 6-1-6-19.

Gilio, M., Scambelluri, M., Agostini, S., Godard, M., Peters, D., Pettke, T., 2019. Petrology and geochemistry of serpentinites associated with the ultra-high pressure Lago di Cignana Unit (Italian Western Alps). J. Petrol. 60 (6), 1229-1262.

Godard, M., Jousselin, D., Bodinier, J.-L., 2000. Relationships between geochemistry and structure beneath a palaeo-spreading Centre: a study of the mantle section in the Oman ophiolite. Earth Planet Sci. Lett. 180, 133-148.

Groppo, C., Castelli, D., 2010. Prograde P-T evolution of a lawsonite eclogite from the Monviso meta-ophiolite (Western Alps): dehydration and redox reactions during subduction of oceanic FeTi-oxide gabbro. J. Petrol. 51, 2489-2514.
Guarnieri, L., Nakamura, E., Piccardo, G.B., Sakaguchi, C., Shimizu, N., Vannucci, R., Zanetti, A., 2012. Petrology, trace element and SR, Nd, Hf isotope geochemistry of the North Lanzo peridotite massif (Western Alps, Italy). J. Petrol. 53, 2259-2306.

Guillot, S., Schwartz, S., Hattori, K., Auzende, A., Lardeaux, J., 2004. The Monviso ophiolitic massif (Western Alps), a section through a serpentinite subduction channel. J. Virtual Explor. 16, 17 (pages).

Guillot, S., Schwartz, S., Reynard, B., Agard, P., Prigent, C., 2015. Tectonic significance of serpentinites. Tectonophysics 646, 1-19.

Hattori, K.H., Guillot, S., 2007. Geochemical character of serpentinites associated with high-to ultrahigh-pressure metamorphic rocks in the Alps, Cuba, and the Himalayas: Recycling of elements in subduction zones. Geochem. Geophys. Geosyst. 8.

Hilairet, N., Reynard, B., Wang. Y., Daniel, I., Merkel, S., Nishiyama, N., Petitgirard, S., 2007. High-pressure creep of serpentine, interseismic deformation, and initiation of subduction. Science 318, 1910-1913.

Ionov, D., Savoyant, L., Dupuy, C., 1992. Application of the ICP/MS technique to trace element anaysis of peridotites and their minerals. Geostand. Geoanal. Res. 16, 311-315.

Jones, C.E., Jenkyns, H.C., 2001. Seawater strontium isotopes, oceanic anoxic events, and seafloor hydrothermal activity in the Jurassic and cretaceous. Am. J. Sci. 301, 112-149.

Kodolányi, J., Pettke, T., 2011. Loss of trace elements from serpentinites during fluidassisted transformation of chrysotile to antigorite-an example from Guatemala. Chem. Geol. 284, 351-362.

Kodolányi, J., Pettke, T., Spandler, C., Kamber, B.S., Gméling, K., 2012. Geochemistry of ocean floor and fore-arc serpentinites: constraints on the ultramafic input to subduction zones. J. Petrol. 53, 235-270.

Lafay, R., Deschamps, F., Schwartz, S., Guillot, S., Godard, M., Debret, B., Nicollet, C., 2013. High-pressure serpentinites, a trap-and-release system controlled by metamorphic conditions: example from the Piedmont zone of the western Alps. Chem. Geol. 343, 38-54.

Li, X.P., Rahn, M., Bucher, K., 2004. Serpentinites of the Zermatt-Saas ophiolite complex and their texture evolution. J. Metamorph. Geol. 22, 159-177.

Liu, L., Sun, Y., Xiao, P., Che, Z., Luo, J., Chen, D., Wang, Y., Zhang, A., Chen, L., Wang, Y., 2002. Discovery of ultrahighpressure magnesite-bearing garnet lherzolite $(>3.8$ GPa) in the Altyn Tagh, Northwest China. Chin. Sci. Bull. 47, 881-886.

Locatelli, M., Verlaguet, A., Agard, P., Federico, L., Angiboust, S., November 2018. Intermediate-depth brecciation along the subduction plate interface (Monviso eclogite, W. Alps). Lithos 320-321, 378-402.

Locatelli, M., Federico, L., Agard, P., Verlaguet, A., 2019. Geology of the southern Monviso metaophiolite complex (W-Alps, Italy). J. Maps 15, 283-297.

Lombardo, B., Nervo, R., Compagnoni, R., Messiga, B., Kienast, J., Mevel, C., Fiora, L., Piccardo, G., Lanza, R., 1978. Osservazioni preliminari sulle ofioliti metamorfiche del Monviso (Alpi Occidentali). Rend. Soc. Ital. Mineral. Petrol. 34, 253-305.

Lombardo, B., Rubatto, D., Castelli, D., 2002. Ion microprobe U-PB dating of zircon from a Monviso metaplagiogranite: Implications for the evolution of the Piedmont-Liguria Tethys in the Western Alps. Ofioliti 27, 109-117.

Lopez Sanchez-Vizcaino, V.L., Trommsdorff, V., Gomez-Pugnaire, M.T., Garrido, C.J., Muntener, O., Connolly, J.A.D., 2005. Petrology of titanian clinohumite and olivine at the high-pressure breakdown of antigorite serpentinite to chlorite harzburgite (Almirez Massif, S. Spain). Contrib. Mineral. Petrol. 149, 627-646.

Luoni, P., Rebay, G., Spalla, M.I., Zanoni, D., 2018. UHP Ti-chondrodite in the Zermatt-Saas serpentinite: Constraints on a new tectonic scenario. Am. Mineral. 103, 1002-1005.

Malaspina, N., Hermann, J., Scambelluri, M., 2009. Fluid/mineral interaction in UHP garnet peridotite. Lithos 107, 38-52

McDonough, W.F., Sun, S.-S., 1995. The composition of the Earth. Chem. Geol. 120, 223-253.

Messiga, B., Kienast, J., Rebay, G., Riccardi, M., Tribuzio, R., 1999. Cr-rich magnesiochloritoid eclogites from the Monviso ophiolites (Western Alps, Italy). J. Metamorph. Geol. 17, 287-300.

Monié, P., 1989. Mise en evidence de l'age eocene moyen du metamorphisme de hautepression dans la nappe ophiolitique du Monviso(Alpes Occidentales)par la methode ${ }^{\wedge}<39>\operatorname{Ar}-{ }^{\wedge}<40>\operatorname{Ar}$. 309. Comptes-Rendues de l'Academie des Science de Paris, pp. $245-251$.

Montes-Hernandez, G., Findling, N., Renard, F., Auzende, A.-L., 2014. Precipitation of ordered dolomite via simultaneous dissolution of calcite and magnesite: New experimental insights into an old precipitation enigma. Cryst. Growth Des. 14, 671-677.

Müntener, O., Pettke, T., Desmurs, L., Meier, M., Schaltegger, U., 2004. Refertilization of mantle peridotite in embryonic ocean basins: trace element and $\mathrm{Nd}$ isotopic evidence and implications for crust-mantle relationships. Earth Planet. Sci. Lett. 221, 293-308.

Niu, Y., 2004. Bulk-rock major and trace element compositions of abyssal peridotites: implications for mantle melting, melt extraction and post-melting processes beneath mid-ocean ridges. J. Petrol. 45, 2423-2458.

Pelletier, L., Müntener, O., 2006. High-pressure metamorphism of the Lanzo peridotite and its oceanic cover, and some consequences for the Sesia-Lanzo zone (northwestern Italian Alps). Lithos 90, 111-130.

Peters, D., Pettke, T., 2017. Evaluation of major to ultra trace element bulk rock chemical analysis of nanoparticulate pressed powder pellets by LA-ICP-MS. Geostand. Geoanal. Res. 41, 5-28.

Peters, D., Bretscher, A., John, T., Scambelluri, M., Pettke, T., 2017. Fluid-mobile elements in serpentinites: Constraints on serpentinisation environments and element cycling in subduction zones. Chem. Geol. 466, 654-666.

Philippot, P., Kienast, J.-R., 1989. Chemical-microstructural changes in eclogite-facies shear zones (Monviso, Western Alps, North Italy) as indicators of strain history and the mechanism and scale of mass transfer. Lithos 23, 179-200.

Philippot, P., Selverstone, J., 1991. Trace-element-rich brines in eclogitic veins: implications for fluid composition and transport during subduction. Contrib. Mineral. Petrol. $106,417-430$. 
Philippot, P., van Roermund, H.L., 1992. Deformation processes in eclogitic rocks: evidence for the rheological delamination of the oceanic crust in deeper levels of subduction zones. J. Struct. Geol. 14, 1059-1077.

Plank, T., 2014. The chemical composition of subducting sediments. Treatise. Geochem. 4, 607-629.

Rampone, E., Romairone, A., Hofmann, A., 2004. Contrasting bulk and mineral chemistry in depleted mantle peridotites: evidence for reactive porous flow. Earth Planet. Sci. Lett. 218, 491-506.

Rehka, M., Hofmann, A., 1997. Recycled ocean crust and sediment in Indian Ocean MORB. Earth Planet. Sci. Lett. 147, 93-106.

Ribeiro, J.M., Lee, C.-T.A., 2017. An imbalance in the deep water cycle at subduction zones: the potential importance of the fore-arc mantle. Earth Planet. Sci. Lett. 479, 298-309.

Rubatto, D., Hermann, J., 2003. Zircon formation during fluid circulation in eclogites (Monviso, Western Alps): implications for $\mathrm{Zr}$ and Hf budget in subduction zones. Geochim. Cosmochim. Acta 67, 2173-2187.

Rudnick, R.L., Gao, S., 2003. Composition of the continental crust. Treatise. Geochem. 3, 659.

Ruh, J.B., Le Pourhiet, L., Agard, P., Burov, E., Gerya, T., 2015. Tectonic slicing of subducting oceanic crust along plate interfaces: numerical modeling. Geochem. Geophys. Geosyst. 16, 3505-3531.

Scambelluri, M., Tonarini, S., 2012. Boron isotope evidence for shallow fluid transfer across subduction zones by serpentinized mantle. Geology 40, 907-910.

Scambelluri, M., Müntener, O., Hermann, J.r., Piccardo, G.B., Trommsdorff, V., 1995. Subduction of water into the mantle: history of an Alpine peridotite. Geology 23, 459-462.

Scambelluri, M., Piccardo, G.B., Philippot, P., Robbiano, A., Negretti, L., 1997. High salinity fluid inclusions formed from recycled seawater in deeply subducted alpine serpentinite. Earth Planet. Sci. Lett. 148, 485-499.

Scambelluri, M., Rampone, E., Piccardo, G.B., 2001. Fluid and element cycling in subducted serpentinite: a trace-element study of the Erro-Tobbio high-pressure ultramafites (Western alps, NW Italy). J. Petrol. 42, 55-67.

Scambelluri, M., Müntener, O., Ottolini, L., Pettke, T.T., Vannucci, R., 2004. The fate of B, Cl and $\mathrm{Li}$ in the subducted oceanic mantle and in the antigorite breakdown fluids. Earth Planet. Sci. Lett. 222, 217-234.

Scambelluri, M., Pettke, T., Rampone, E., Godard, M., Reusser, E., 2014. Petrology and trace element budgets of high-pressure peridotites indicate subduction dehydration of serpentinized mantle (Cima di Gagnone, Central Alps, Switzerland). J. Petrol. 55, 459-498.
Scambelluri, M., Pettke, T., Cannaò, E., 2015. Fluid-related inclusions in Alpine highpressure peridotite reveal trace element recycling during subduction-zone dehydration of serpentinized mantle (Cima di Gagnone, Swiss Alps). Earth Planet. Sci. Lett. 429, 45-59.

Scambelluri, M., Bebout, G.E., Belmonte, D., Gilio, M., Campomenosi, N., Collins, N. Crispini, L., 2016. Carbonation of subduction-zone serpentinite (high-pressure ophicarbonate; Ligurian Western Alps) and implications for the deep carbon cycling. Earth Planet. Sci. Lett. 441, 155-166.

Scambelluri, M., Cannaò, E., Gilio, M., 2019. The water and fluid-mobile element cycles during serpentinite subduction. A review. Eur. J. Mineral. 31 (3), 405-428.

Schwartz, S., Lardeaux, J.-M., Guillot, S., Tricart, P., 2000. Diversité du métamorphisme éclogitique dans le massif ophiolitique du Monviso (Alpes occidentales, Italie). Geodin. Acta 13, 169-188.

Schwartz, S., Allemand, P., Guillot, S., 2001. Numerical model of the effect of serpentinites on the exhumation of eclogitic rocks: insights from the Monviso ophiolitic massif (Western Alps). Tectonophysics 342, 193-206.

Schwartz, S., Guillot, S., Reynard, B., Lafay, R., Debret, B., Nicollet, C., Lanari, P., Auzende, A.L., 2013. Pressure-temperature estimates of the lizardite/antigorite transition in high pressure serpentinites. Lithos 178, 197-210.

Shen, T., Hermann, J., Zhang, L., Lü, Z., Padrón-Navarta, J.A., Xia, B., Bader, T., 2015. UHP metamorphism documented in Ti-chondrodite-and Ti-clinohumite-bearing serpentinized ultramafic rocks from Chinese southwestern Tianshan. J. Petrol. 56 1425-1458.

Spandler, C., Pettke, T., Rubatto, D., 2011. Internal and external fluid sources for eclogitefacies veins in the Monviso meta-ophiolite, Western Alps: implications for fluid flow in subduction zones. J. Petrol. 52, 1207-1236.

Van Keken, P.E., Kiefer, B., Peacock, S.M., 2002. High-resolution models of subduction zones: Implications for mineral dehydration reactions and the transport of water into the deep mantle. Geochem. Geophys. Geosyst. 3.

Wada, I., Wang, K., 2009. Common depth of slab-mantle decoupling: Reconciling diversity and uniformity of subduction zones. Geochem. Geophys. Geosyst. 10.

Watanabe, T., Shirasugi, Y., Yano, H., Michibayashi, K., 2011. Seismic velocity in antigoritebearing serpentinite mylonites. Geol. Soc. Lond. Spec. Publ. 360, 97-112.

White, W., Klein, E., 2014. 4.13-Composition of the Oceanic Crust. Treatise on Geochemistry, Second edition. 